\title{
Long-time Asymptotics with Geometric Singularities in the Spatial Variables
}

\author{
T. Krainer* and B.-W. Schulze ${ }^{\dagger}$
}

\section{Contents}

Introduction

1 Anisotropic operators in a cylinder with a conical base 3

1.1 Manifolds with conical singularities and operators of Fuchs type . . . . . . . 3

1.2 Typical operators and symbol structures . . . . . . . . . . . . . 3

2 Weighted wedge Sobolev spaces and edge asymptotics 6

2.1 Discrete edge asymptotics . . . . . . . . . . . . . . . 6

2.2 Continuous edge asymptotics with discrete limit at infinity . . . . . . . 8

2.3 Calculus with operator-valued symbols . . . . . . . . . . . . . . 11

3 Corner asymptotics at infinity 12

3.1 The structure of singular functions . . . . . . . . . . . . . . . 12

3.2 Operators with trace and potential conditions . . . . . . . . . . . 13

3.3 Asymptotics and (anisotropic) elliptic regularity . . . . . . . . . . . . 16

References

\section{Introduction}

Parabolic equations (or, more generally, anisotropic elliptic equations) in an infinite spacetime cylinder $M \times \mathbb{R}$ may be studied under the aspect of operators on a non-compact configuration with geometric singularities. This concerns, in particular, the construction of parametrices within an anisotropic calculus of pseudo-differential operators and the characterisation of asymptotics of solutions.

We consider the case that the cross-section $M$ of the cylinder has conical singularities, i.e., $M \times \mathbb{R}$ has edges (if $S$ is the finite set of conical points of $M$, then $Y:=S \times \mathbb{R}$ is the system of edges in time direction). Let $r \in \mathbb{R}_{+}$denote the distance variable of a point of $M \times \mathbb{R}$ to such an edge (i.e., $r$ is the cone axis variable for a conical singularity of $M$ ). In this paper we shall show (under some natural conditions on the $t$-dependence of the coefficients of the operators) that $t=\infty$ can be interpreted as an "anisotropic" corner (in the sense of a corner singularity, cf. the definitions below) and that long-time asymptotics have the character of multiple edge-corner asymptotics with the time $t$ as the corner axis and $r$ as a second spatial axial variable.

\footnotetext{
* Supported by Deutsche Forschungsgemeinschaft

†Supported by the EU Research and Training Network "Geometric Analysis"
} 
Ellipticity (especially the anisotropic one) on a manifold with geometric singularities (say, of conical, edge or corner type) is described by a hierarchy of principal symbols, where the (scalar) interior symbols come from the operators themselves, while a further tuple of operator-valued symbols is induced by the singularities. Examples are the conormal symbols near conical singularities, cf. Kondrat'ev [5], Schulze [16], or the boundary symbols in boundary value problems, cf. Boutet de Monvel [2], Rempel and Schulze [10]. While for conical singularities the ellipticity with respect to conormal symbols is connected with a global spectral information along the base of the cone (with eigenvalues non-linearly involved as complex variables in meromorphic operator functions), for boundary value problems the ellipticity of boundary symbols encodes the nature of additional conditions of trace and potential type on the boundary.

Similarly, for edge singularities we have operator-valued edge symbols containing (in general) the symbols of edge conditions with an analogue of the Shapiro-Lopatinskij condition in the elliptic case, cf. Schulze [13], [19].

The nature of trace and potential symbols along an edge $Y$ is connected with the singular functions of asymptotics near $Y$ with respect to $r \in \mathbb{R}_{+}$. Considering a (stretched) wedge $\mathbb{R}_{+} \times X \times Y$ with a (stretched) model cone $\mathbb{R}_{+} \times X \ni(r, x)$, where $X$ is a closed, compact $C^{\infty}$ manifold, asymptotics of solutions $u(r, x, t)$ to elliptic edge problems may have the following form:

$$
u(r, x, t) \sim \sum_{j=0}^{\infty} \sum_{k=0}^{m_{j}(t)} c_{j k}(x, t) r^{-p_{j}(t)} \log ^{k} r
$$

for $r \rightarrow 0$, where $p_{j}(t) \in \mathbb{C}, \operatorname{Re} p_{j}(t) \rightarrow-\infty$ as $j \rightarrow \infty$ for every fixed $t \in Y$, and coefficients $c_{j k}(x, t)$ that are smooth in $x \in X$. (Asymptotics of the form (0.0.1) for fixed $t$ are also called discrete, cf. Section 2.1 below.)

(0.0.1) is true, indeed, as soon as $u$ is assumed to be smooth outside the edge, cf. Schulze [12] (for the isotropic case). However, if $u$ is of some finite Sobolev smoothness, the coefficients have a rather complicated structure with respect to $t$; their Sobolev smoothness depends on $\operatorname{Re} p_{j}(t)$, and there is an extra dependence on $t$. The exponents may be variable under varying $t$ and form in general rather irregular clouds of points in the complex plane. Both the adequate description of $t$-wise asymptotics themselves and the understanding of regularity of coefficients belong to the essential features of corner asymptotics.

Variable discrete asymptotics can be managed with the help of continuous asymptotics as have been introduced in Schulze [11] (formulated in terms of vector-valued analytic functionals in the complex plane). In fact, continuous asymptotics seem to be indispensable to understanding variable discrete edge asymptotics in reasonable generality. In the present paper we mainly discuss asymptotics for $t \rightarrow \infty$ with discrete limit and formulate asymptotics for finite $t$ as continuous ones without specifying the pointwise discrete behaviour for finite $t$ that would be an extra program.

Another ingredient is a global contribution from the principal conormal symbol at infinity, again with non-linearly involved eigenvalues, now related to the base of the corner with its conical singularities. The interplay between wedge and global corner conormal effects has been characterised in Schulze [15] within a corresponding corner pseudo-differential calculus, cf. also [21]. In the present paper we extend these results in combination with the main idea of a new characterisation of long-time asymptotics for (non-autonomous) parabolic (pseudodifferential) operators of Krainer and Schulze [7] for a smooth space-time cylinder, under rather general assumptions on the $t$-dependence of coefficients at infinity (asymptotics of a similar type also hold for parabolic boundary value problems, cf. Krainer [6]). Asymptotics in the case of conical singularities in spatial variables may also be asked for boundary value problems, i.e., when $M$ is a manifold with boundary and conical singularities; this 
is much more complicated and requires the results of [22]. In the present paper we try to minimise technicalities and therefore consider the case without boundary. We may look at typical differential operators, though we get the results in general for pseudo-differential operators. Elements of the technique on anisotropic pseudo-differential operators near edgesingularities may also be found in Buchholz and Schulze [3].

\section{Anisotropic operators in a cylinder with a conical base}

The natural anisotropic differential operators on a space-time cylinder with a singular cross section are degenerate in a typical way (in stretched coordinates). They have a scalar anisotropic homogeneous principal symbol, and the geometric singularities generate a further (operator-valued) principal edge symbol as well as a subordinate principal conormal symbol.

\subsection{Manifolds with conical singularities and operators of Fuchs type}

Let $M$ be a compact manifold with a finite set $S \subset M$ of conical singularities, i.e., $M \backslash S$ is smooth, and every $v \in S$ has a neighbourhood $V$ in $M$ that is homeomorphic to a cone $X^{\Delta}:=\left(\overline{\mathbb{R}}_{+} \times X\right) /(\{0\} \times X)$ for a smooth compact and closed manifold $X=X(v)$, such that $V \backslash\{v\}$ is diffeomorphic to the open (stretched) cone $X^{\wedge}:=\mathbb{R}_{+} \times X$. The map $V \rightarrow X^{\Delta}$ is regarded as a "singular chart" on $M$ near $v$, and we fix an atlas such that for two such charts the transition map restricted to $X^{\wedge}$ extends to a diffeomorphism of $\overline{\mathbb{R}}_{+} \times X$ to itself, i.e., has a smooth extension up to $r=0$. Thus, with $M$ we can associate the stretched manifold $\mathbb{M}$ that is a smooth, compact manifold with boundary $\partial \mathbb{M} \cong \bigcup_{v \in S} X(v)$. Conversely, to every smooth, compact manifold $\mathbb{M}$ with boundary we find a manifold $M$ with conical singularities $S$ as the image under the quotient map $\mathbb{M} \rightarrow M$ that is a diffeomorphism int $\mathbb{M} \rightarrow M \backslash S$, where every component of $\partial M$ is mapped to an element $v \in S$.

For simplicity, throughout this paper we assume that $M$ has only one conical singularity $\{v\}$; the considerations for an arbitrary finite number of conical singularities are completely analogous.

Let $\operatorname{Diff}^{\nu}(X)$ denote the space of all differential operators of order $\nu$ on $X$ (with smooth coefficients); this is a Fréchet space in a natural way. Moreover, define $\operatorname{Diff}_{\text {Fuchs }}^{\mu}(\mathbb{M})$ to be the space of all differential operators of order $\mu$ on int $\mathbb{M}$ (with smooth coefficients) that have locally near $\partial \mathbb{M}=X$ in the splitting of variables $(r, x) \in X^{\wedge}$ the form

$$
r^{-\mu} \sum_{j=0}^{\mu} a_{j}(r)\left(-r \frac{\partial}{\partial r}\right)^{j}
$$

with operator-valued coefficients $a_{j} \in C^{\infty}\left(\overline{\mathbb{R}}_{+}, \operatorname{Diff}^{\mu-j}(X)\right), j=0, \ldots, \mu$. Operators of that form may be regarded as the typical ones on a manifold with conical singularities and are called of Fuchs type. Note, that when $g_{\mathbb{M}}$ is a Riemannian metric on $\mathbb{M}$ that is locally near $\partial \mathbb{M}$ of the form $d r^{2}+r^{2} g_{X}(r)$ for an $r$-dependent family $g_{X}(r)$ of Riemannian metrics on $X$, smooth in $r$ up to $r=0$, the associated Laplace-Beltrami operator $\Delta_{g_{\mathbb{M}}}$ is of Fuchs type in this sense (of order $\mu=2$ ).

\section{$1.2 \quad$ Typical operators and symbol structures}

The simplest example of operators on $\mathbb{M} \times \mathbb{R}$ that we have in mind is the heat operator $\partial_{t}-\Delta_{g_{\mathbb{M}}}$. More generally, we may admit $g_{\mathbb{M}}$ smoothly depending on $t$. 
The differential operators on $\mathbb{M} \times \mathbb{R}$ in general are assumed to be of the form

$$
A=\sum_{k=0}^{\mu / \ell} A_{k}(t) D_{t}^{k}
$$

for certain $A_{k}(t) \in C^{\infty}\left(\mathbb{R}, \operatorname{Diff}_{\text {Fuchs }}^{\mu-k \ell}(\mathbb{M})\right)$ with a dependence on $t$ for $t \rightarrow \infty$ that will be specified below. $\ell \in \mathbb{N}$ is a chosen anisotropy (that equals 2 for the heat operator) such that $\mu / \ell \in \mathbb{N}$.

The presence of a geometric singularity (here, a conical one) in the spatial variables induces an enormous variety of new structures, compared with the smooth case, even if we are only interested in local aspects with respect to $t$, e.g., (pseudo-differential) parametrices along the time-edge or asymptotics of solutions. The question of long-time asymptotics (under our assumptions on the coefficients for $t \rightarrow \infty$ ) is connected with the fact that the configuration $M \times \mathbb{R}$ for $t \rightarrow \infty$ has the character of an "anisotropic" corner, i.e., a cone with a base that has itself conical singularities.

A transparent description of asymptotic phenomena requires techniques of the pseudodifferential analysis on singular manifolds that have edge and corner singularities, here, in an anisotropic set-up. Asymptotics of solutions will be obtained in form of some anisotropic elliptic regularity at infinity, based on the properties of parametrices in spaces with asymptotics. In this context it is natural to embed our concrete operators into an algebra of pseudo-differential operators, connected with the choice of $\ell \in \mathbb{N}$.

Inserting for $A_{k}(t)$ in (1.2.1) the local expressions $r^{-\mu+k \ell} \sum_{j=0}^{\mu-k \ell} a_{j k}(r, t)\left(-r \frac{\partial}{\partial r}\right)^{j}$, $a_{j k}(r, t) \in C^{\infty}\left(\overline{\mathbb{R}}_{+} \times \mathbb{R}, \operatorname{Diff}^{\mu-k \ell-j}(X)\right)$, cf. formula (1.1.1), we get

$$
A=r^{-\mu} \sum_{k=0}^{\mu / \ell} \sum_{j=0}^{\mu-k \ell} a_{j k}(r, t)\left(-r \frac{\partial}{\partial r}\right)^{j} r^{k \ell} D_{t}^{k} .
$$

This has the shape of an (anisotropic) edge-degenerate differential operator, cf. the terminology of [19], Section 3.1.2. In other words, $t$ is treated as a variable along the edge.

Our considerations will depend on a suitable notion of anisotropic ellipticity (not really parabolicity that would be a stronger condition). To this end we introduce the tuple $\sigma(A)=$ $\left(\sigma_{\psi}(A), \sigma_{\wedge}(A)\right)$ of principal symbols. $\sigma_{\psi}(A)$ is simply the homogeneous principal symbol of $A$, locally being a smooth function $\sigma_{\psi}(A)(\widetilde{x}, t, \widetilde{\xi}, \tau)$ in the variables $(\widetilde{x}, t) \in \operatorname{int} \mathbb{M} \times \mathbb{R}$ with covariables $(\widetilde{\xi}, \tau) \neq 0$, satisfying

$$
\sigma_{\psi}(A)\left(\widetilde{x}, t, \lambda \widetilde{\xi}, \lambda^{\ell} \tau\right)=\lambda^{\mu} \sigma_{\psi}(A)(\widetilde{x}, t, \widetilde{\xi}, \tau)
$$

for all $\lambda \in \mathbb{R}_{+}$. The second component, the so-called homogeneous principal edge symbol $\sigma_{\wedge}(A)(t, \tau)$, is the typical contribution from the edge singularity. It is an operator family parametrised by $(t, \tau) \in \mathbb{R} \times(\mathbb{R} \backslash\{0\})$

$$
\sigma_{\wedge}(A)(t, \tau): \mathcal{K}^{s, \gamma}\left(X^{\wedge}\right) \rightarrow \mathcal{K}^{s-\mu, \gamma-\mu}\left(X^{\wedge}\right),
$$

acting between weighted Sobolev spaces $\mathcal{K}^{s, \gamma}\left(X^{\wedge}\right)$ on the infinite stretched cone $X^{\wedge}$, of smoothness $s \in \mathbb{R}$ and weight $\gamma \in \mathbb{R}$, cf. [19], Definition 2.1.57. Recall that the spaces $\mathcal{K}^{s, \gamma}\left(X^{\wedge}\right)$ are based on the Mellin transform with respect to $r \in \mathbb{R}_{+}$near zero, otherwise on the Fourier transform as usual, and they equal the standard Sobolev spaces for $r \rightarrow \infty$. If $\omega(r)$ is a cut-off function near $r=0$ (i.e., any element in $C_{0}^{\infty}\left(\overline{\mathbb{R}}_{+}\right)$such that $\omega(r)=1$ near $r=0$ ) we have $\omega r^{\varrho} \mathcal{K}^{s, \gamma}\left(X^{\wedge}\right)=\omega \mathcal{K}^{s, \gamma+\varrho}\left(X^{\wedge}\right)$ for arbitrary $s, \gamma, \varrho \in \mathbb{R}$. Moreover, $\mathcal{K}^{0,0}\left(X^{\wedge}\right)=r^{-\frac{n}{2}} L^{2}\left(\mathbb{R}_{+} \times X\right)$ for $n=\operatorname{dim} X$, where the $L^{2}$ space refers to $d r d x$, with $d x$ being the measure associated with a fixed Riemannian metric on $X$. For $u(r, x) \in \mathcal{K}^{s, \gamma}\left(X^{\wedge}\right)$ 
we have $\left(\kappa_{\lambda} u\right)(r, x):=\lambda^{\frac{n+1}{2}} u(\lambda r, x) \in \mathcal{K}^{s, \gamma}\left(X^{\wedge}\right)$ for every $\lambda \in \mathbb{R}_{+}$, and $\kappa_{\lambda}: \mathcal{K}^{s, \gamma}\left(X^{\wedge}\right) \rightarrow$ $\mathcal{K}^{s, \gamma}\left(X^{\wedge}\right)$ is a strongly continuous group of isomorphisms on $\mathcal{K}^{s, \gamma}\left(X^{\wedge}\right)$ for every $s, \gamma$ (that is unitary when $s=\gamma=0$ ).

We set

$$
\sigma_{\wedge}(A)(t, \tau)=r^{-\mu} \sum_{k=0}^{\mu / \ell} \sum_{j=0}^{\mu-k \ell} a_{j k}(0, t)\left(-r \frac{\partial}{\partial r}\right)^{j} r^{k \ell} \tau .
$$

Then

$$
\sigma_{\wedge}(A)\left(t, \lambda^{\ell} \tau\right)=\lambda^{\mu} \kappa_{\lambda} \sigma_{\wedge}(A)(t, \tau) \kappa_{\lambda}^{-1}
$$

for all $(t, \tau) \in \mathbb{R} \times(\mathbb{R} \backslash\{0\}), \lambda \in \mathbb{R}_{+}$.

The operator $A$ is called anisotropic elliptic with respect to $\sigma_{\psi}(A)$ if $\sigma_{\psi}(A)(\widetilde{x}, t, \widetilde{\xi}, \tau) \neq 0$ for all $(\widetilde{x}, t, \widetilde{\xi}, \tau) \in T^{*}($ int $\mathbb{M} \times \mathbb{R}) \backslash 0$ and if in addition locally near $\partial \mathbb{M}$ in a splitting of variables $(r, x, t, \varrho, \xi, \tau)$

$$
\widetilde{\sigma}_{\psi}(A)(r, x, t, \varrho, \xi, \tau):=r^{\mu} \sigma_{\psi}(A)\left(r, x, t, r^{-1} \varrho, \xi, r^{-\ell} \tau\right) \neq 0
$$

for all $(r, x, t, \varrho, \xi, \tau) \in T^{*}([0, \varepsilon) \times X \times \mathbb{R}) \backslash 0$ for some $\varepsilon>0$. The latter condition is invariant, i.e., independent of the choice of a singular chart near the conical singularity.

Let us set $\Gamma_{\beta}=\{z \in \mathbb{C}: \operatorname{Re} z=\beta\}$ for any real $\beta$.

Theorem 1.2.1 Let $A$ be anisotropic elliptic with respect to $\sigma_{\psi}$. Then for every $t \in \mathbb{R}$ there is a countable discrete set $D(t) \subset \mathbb{C}$ such that $D(t) \cap\left\{z \in \mathbb{C}: c \leq \operatorname{Re} z \leq c^{\prime}\right\}$ is finite for every $c \leq c^{\prime}$, such that (1.2.3) is a Fredholm operator for every $\gamma \in \mathbb{R}$ with the property $\Gamma_{\frac{n+1}{2}-\gamma} \cap D(t)=\emptyset$, for arbitrary $(t, \tau) \in \mathbb{R} \times(\mathbb{R} \backslash\{0\})$, and for all $s \in \mathbb{R}$.

This type of result is known in analogous form from elliptic operator-valued edge symbols, cf. [19], Theorem 3.5.1, and the exceptional set $D(t)$ is characterised in the following remark:

Remark 1.2.2 The operator family

$$
\sigma_{M}(A)(t, z):=\sum_{j=0}^{\mu} a_{j 0}(0, t) z^{j}: H^{s}(X) \rightarrow H^{s-\mu}(X),
$$

called the principal conormal symbol of the operator $A$, is holomorphic in $z \in \mathbb{C}$ for every $t \in \mathbb{R}$. The anisotropic $\sigma_{\psi}$-ellipticity of $A$ implies that (1.2.6) is a family of Fredholm operators that is bijective for all $z \in \mathbb{C} \backslash D(t)$. In other words, $D(t)$ is determined by the non-bijectivity points of (1.2.6).

Remark 1.2.3 Under the conditions of Theorem 1.2.1 the dimensions of $\operatorname{ker} \sigma_{\wedge}(A)(t, \tau)$ and $\operatorname{coker} \sigma_{\wedge}(A)(t, \tau)$ are independent of $s \in \mathbb{R}$. Thus, also the index ind $\sigma_{\wedge}(A)(t, \tau)$ is independent of $s$, though it may depend on the weight $\gamma$.

We study operators $A$ under the following hypotheses:

(i) $A$ is anisotropic $\sigma_{\psi}$-elliptic.

(ii) There is a $T \in \mathbb{R}$ and a $\gamma \in \mathbb{R}$ such that $\Gamma_{\frac{n+1}{2}} \cap D(t)=\emptyset$ for all $t \geq T$.

(iii) ind $\sigma_{\wedge}(A)(t, \tau)=$ ind $\sigma_{\wedge}(A)(t,-\tau)$ for all $t \geq T, \tau \neq 0$. 
(iv) $A$ has smooth coefficients up to $t= \pm \infty$ in the sense that when we represent $A$ in the form $\sum_{\alpha, k} \widetilde{a}_{\alpha k}(\widetilde{x}, t) D_{\widetilde{x}}^{\alpha} D_{t}^{k}$ locally with respect to $\widetilde{x}$ in a coordinate neighbourhood $U \subset \operatorname{int} \mathbb{M}$, we have $\widetilde{a}_{\alpha k}(\widetilde{x}, \mp \log \widetilde{t}) \in C^{\infty}\left(U \times \overline{\mathbb{R}}_{+}\right)$for all $\alpha, k$, and in addition $a_{j k}(r, \mp \log \widetilde{t}) \in C^{\infty}\left(\overline{\mathbb{R}}_{+} \times \overline{\mathbb{R}}_{+}, \operatorname{Diff}^{\mu-k \ell-j}(X)\right)$ for all $j, k$, cf. formula (1.2.2).

It can be proved that condition (iii) is independent of the choice of $\gamma$. Concerning (iv), only the behaviour for $t \rightarrow \infty$ is of interest. The analogous conditions on the coefficients for $t \rightarrow-\infty$ is imposed without loss of generality; it will be convenient to formulate results in Sobolev spaces globally in $t \in \mathbb{R}$ and to avoid cut-offs with respect to $t=\infty$.

\section{Weighted wedge Sobolev spaces and edge asymptotics}

Asymptotics of solutions in the infinite space-time cylinder are characterised by a specific interaction between edge asymptotics for finite $t$ and the long-time behaviour. The functional analytic structure of singular functions is non-trivial even for equations with constant coefficients; in this case constant discrete edge asymptotics are an adequate choice. For general $t$-dependence of coefficients we need continuous asymptotics, a generalisation of discrete ones.

\subsection{Discrete edge asymptotics}

Asymptotics for $t \rightarrow \infty$ in our (stretched) space-time cylinder $\mathbb{M} \times \mathbb{R}$ will contain contributions from the edge and from the base of the corner at infinity. The edge part of the asymptotics is non-trivial also in finite time intervals and necessary for understanding the limit behaviour for $t \rightarrow \infty$. In this section we describe the functional analytic structure of the corresponding singular functions in terms of anisotropic wedge Sobolev spaces.

Let $E$ be a Hilbert space and $\kappa_{\lambda}: E \rightarrow E, \lambda \in \mathbb{R}_{+}$, be a strongly continuous group of isomorphisms. Then $\mathcal{W}^{s}(\mathbb{R}, E)_{(\ell)}, s \in \mathbb{R}$, is defined to be the completion of $\mathcal{S}(\mathbb{R}, E)$ with respect to the norm $\left\{\int\langle\tau\rangle_{\ell}^{2 s}\left\|\kappa_{\langle\tau\rangle_{\ell}}^{-1} \hat{u}(\tau)\right\|_{E}^{2} d \tau\right\}^{\frac{1}{2}}, s \in \mathbb{R}$, where $\langle\tau\rangle_{\ell}=\left(1+|\tau|^{2}\right)^{\frac{1}{2 \ell}}$, and $\mathcal{S}(\mathbb{R}, E)$ is the Schwartz space of $E$-valued functions. (Clearly, for $\mathcal{W}^{s}(\mathbb{R}, E):=\mathcal{W}^{s}(\mathbb{R}, E)_{(1)}$, we have $\mathcal{W}^{s}(\mathbb{R}, E)_{(\ell)}=\mathcal{W}^{s / \ell}(\mathbb{R}, E)$.) Moreover, if $E$ is a Fréchet space, written as a projective limit

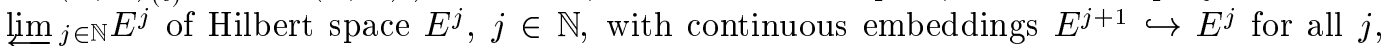
and if $\left\{\kappa_{\lambda}\right\}_{\lambda \in \mathbb{R}_{+}}$is a strongly continuous group of isomorphisms on $E^{0}$ that restricts to a strongly continuous group of isomorphisms on $E^{j}$ for all $j$, we say that $E$ is equipped with a group action.

We then have continuous embeddings $\mathcal{W}^{s}\left(\mathbb{R}, E^{j+1}\right)_{(\ell)} \hookrightarrow \mathcal{W}^{s}\left(\mathbb{R}, E^{j}\right)_{(\ell)}$ for all $j$, and we write $\mathcal{W}^{s}(\mathbb{R}, E)_{(\ell)}=\lim _{j \in \mathbb{N}} \mathcal{W}^{s}\left(\mathbb{R}, E^{j}\right)_{(\ell)}$.

The Hilbert space $\overleftarrow{E}=\mathcal{K}^{s, \gamma}\left(X^{\wedge}\right)$, endowed with the group action $\left\{\kappa_{\lambda}\right\}_{\lambda \in \mathbb{R}_{+}},\left(\kappa_{\lambda} u\right)(r, x)=$ $\lambda^{\frac{n+1}{2}} u(\lambda r, x)$, gives rise to the weighted wedge Sobolev space

$$
\mathcal{W}^{s, \gamma}\left(X^{\wedge} \times \mathbb{R}\right)_{(\ell)}:=\mathcal{W}^{s}\left(\mathbb{R}, \mathcal{K}^{s, \gamma}\left(X^{\wedge}\right)\right)_{(\ell)} .
$$

For every $-\infty<\vartheta<0$ and $\Theta=(\vartheta, 0]$ we have the Fréchet space

$$
\mathcal{K}_{\Theta}^{s, \gamma}\left(X^{\wedge}\right)=\lim _{\varepsilon>0} \mathcal{K}^{s, \gamma-\vartheta-\varepsilon}\left(X^{\wedge}\right)
$$

of functions of flatness $\Theta$ (relative to the weight $\gamma$ near $r=0$ ). Using the group action on $\mathcal{K}_{\Theta}^{s, \gamma}\left(X^{\wedge}\right)$ induced by $\mathcal{K}^{s, \gamma}\left(X^{\wedge}\right)$ we then get an associated space

$$
\mathcal{W}_{\Theta}^{s, \gamma}\left(X^{\wedge} \times \mathbb{R}\right)_{(\ell)}:=\mathcal{W}^{s}\left(\mathbb{R}, \mathcal{K}_{\Theta}^{s, \gamma}\left(X^{\wedge}\right)\right)_{(\ell)}
$$

of functions of edge flatness $\Theta$ (relative to $\gamma$ ) near $r=0$. 
The asymptotics themselves are defined as sums of singular and flat functions. To define corresponding function spaces including their adequate Fréchet topologies we employ the following notions. Given Fréchet spaces $E_{0}$ and $E_{1}$, embedded in a Hausdorff topological vector space $H$, we write $E_{0}+E_{1}=\left\{e_{0}+e_{1}: e_{0} \in E_{0}, e_{1} \in E_{1}\right\}$ and endow this space with the Fréchet topology induced by the bijection $E_{0}+E_{1} \cong E_{0} \oplus E_{1} / \Delta$ for $\Delta=\{(e,-e)$ : $\left.e \in E_{0} \cap E_{1}\right\}$. We will call $E_{0}+E_{1}$ the non-direct sum of $E_{0}$ and $E_{1}$. Moreover, if a Fréchet space $E$ is a (left) module over an algebra $A$, for any $a \in A$ we define $[a] E$ to be the completion of $\{a e: e \in E\}$ in $E$.

In this paper a cut-off function is an element $\omega(r) \in C_{0}^{\infty}\left(\overline{\mathbb{R}}_{+}\right)$with $\omega(r)=1$ in a neighbourhood of $r=0$.

Asymptotics of elements $u(r, x) \in \mathcal{K}^{s, \gamma}\left(X^{\wedge}\right)$ for $r \rightarrow 0$ are in the simplest case discrete, i.e., of the form

$$
u(r, x)=\omega(r) \sum_{j=0}^{N} \sum_{k=0}^{m_{j}} c_{j k}(x) r^{-p_{j}} \log ^{k} r+u_{\text {flat }}(r, x)
$$

for a cut-off function $\omega$, a flat remainder $u_{\text {flat }} \in \mathcal{K}_{\Theta}^{s, \gamma}\left(X^{\wedge}\right)$, and a sequence of pairs $\left(p_{j}, m_{j}\right) \in$ $\mathbb{C} \times \mathbb{N}$, where $\frac{n+1}{2}-\gamma+\vartheta<\operatorname{Re} p_{j}<\frac{n+1}{2}-\gamma$ for all $j=0, \ldots, N$. The coefficients $c_{j k}$ are assumed to belong to finite-dimensional subspaces $L_{j} \subset C^{\infty}(X)$ for $0 \leq k \leq m_{j}$. Given weight data $(\gamma, \Theta)$ we call such a sequence $P=\left\{\left(p_{j}, m_{j}, L_{j}\right)\right\}$ a discrete asymptotic type. For $\Theta=(-\infty, 0]$ we admit infinite sequences $P$ such that $\operatorname{Re} p_{j} \rightarrow-\infty$ as $j \rightarrow \infty$. Let $\pi_{\mathbb{C}} P$ be the union of all $p_{j}$. Let $\mathcal{K}_{P}^{s, \gamma}\left(X^{\wedge}\right)$ (first for finite $\Theta$ ) denote the subspace of all $u \in \mathcal{K}^{s, \gamma}\left(X^{\wedge}\right)$ of the form (2.1.2) for a given $P$. The space $\mathcal{K}_{P}^{s, \gamma}\left(X^{\wedge}\right)$ is Fréchet in a natural way (in fact, $\mathcal{K}_{P}^{s, \gamma}\left(X^{\wedge}\right) \cong \mathcal{K}_{\Theta}^{s, \gamma}\left(X^{\wedge}\right)+\mathcal{E}_{P}\left(X^{\wedge}\right)$, where $\mathcal{E}_{P}\left(X^{\wedge}\right)$ is defined to be the linear span of all involved singular functions $\omega(r) c_{j k}(x) r^{-p_{j}} \log ^{k} r$ which is of finite dimension). For infinite $\Theta$ and $P$ we can define $\mathcal{K}_{P}^{s, \gamma}\left(X^{\wedge}\right)$ by a simple projective limit over corresponding spaces for finite weight intervals. The space $\mathcal{K}_{P}^{s, \gamma}\left(X^{\wedge}\right)$ has a group action, induced by that on $\mathcal{K}^{s, \gamma}\left(X^{\wedge}\right)$. This gives us the space

$$
\mathcal{W}_{P}^{s, \gamma}\left(X^{\wedge} \times \mathbb{R}\right)_{(\ell)}:=\mathcal{W}^{s}\left(\mathbb{R}, \mathcal{K}_{P}^{s, \gamma}\left(X^{\wedge}\right)\right)_{(\ell)}
$$

of elements of discrete edge asymptotics of type $P$.

Remark 2.1.1 Let $P=\left\{\left(p_{j}, m_{j}, L_{j}\right)\right\}_{j=0, \ldots, N}$ be a discrete asymptotic type, associated with weight data $(\gamma, \Theta), \Theta=(\vartheta, 0]$ finite. Then $\mathcal{W}_{P}^{s, \gamma}\left(X^{\wedge} \times \mathbb{R}\right)_{(\ell)}$ is characterised as the subspace of all $u(r, x, t) \in \mathcal{W}^{s, \gamma}\left(X^{\wedge} \times \mathbb{R}\right)_{(\ell)}$ such that for a choice of a cut-off function $\omega(r)$

$$
\begin{aligned}
u(r, x, t)=\sum_{j=0}^{N} \sum_{k=0}^{m_{j}} F_{\tau \rightarrow t}^{-1}\left\{[\tau]_{\ell}^{\frac{n+1}{2}} \omega(r[\tau] \ell) c_{j k}(x)\left(r[\tau]_{\ell}\right)^{-p_{j}} \log ^{k}\left(r[\tau]_{\ell}\right) \hat{v}_{j k}(\tau)\right\} & \\
& +u_{\Theta}(r, x, t)
\end{aligned}
$$

where $c_{j k} \in L_{j}, v_{j k} \in H^{s / \ell}(\mathbb{R}), 0 \leq k \leq m_{j}, j=0, \ldots, N$ and $u_{\Theta}(r, x, t) \in \mathcal{W}_{\Theta}^{s, \gamma}\left(X^{\wedge} \times \mathbb{R}\right)_{(\ell)}$. For $P=\left\{\left(p_{j}, m_{j}, L_{j}\right)\right\}_{j \in \mathbb{N}}$ associated with $(\gamma,(-\infty, 0])$ the space $\mathcal{W}_{P}^{s, \gamma}\left(X^{\wedge} \times \mathbb{R}\right)_{(\ell)}$ consists of all $u(r, x, t) \in \mathcal{W}^{s, \gamma}\left(X^{\wedge} \times \mathbb{R}\right)_{(\ell)}$ such that for every finite $\Theta=(\vartheta, 0]$ we have a representation of the form (2.1.4), where the numeration of the points of $\pi_{\mathbb{C}} P$ is chosen in such a way that $\left\{p_{0}, \ldots, p_{N}\right\}=\pi_{\mathbb{C}} P \cap\left\{z: \frac{n+1}{2}-\gamma+\vartheta<\operatorname{Re} z<\frac{n+1}{2}-\gamma\right\}$.

Remark 2.1.2 The expansion (2.1.4) can also be interpreted in terms of particular analytic functionals $\zeta \in \mathcal{A}^{\prime}\left(\mathbb{C}, C^{\infty}\left(X, \hat{H}^{s / \ell}(\mathbb{R})\right)\right)$ carried by the set $\left\{p_{0}, \ldots, p_{N}\right\}, \hat{H}^{s / \ell}(\mathbb{R})=$ $F_{t \rightarrow \tau} H^{s / \ell}(\mathbb{R})$ (cf. also the notation in Section 2.2 below). In fact, setting

$$
\langle\zeta, h\rangle=\left.\sum_{j=0}^{N} \sum_{k=0}^{m_{j}}(-1)^{k} c_{j k}(x) \hat{v}_{j k}(\tau)\left(\frac{d^{k}}{d z^{k}} h(z)\right)\right|_{z=p_{j}},
$$


$h \in \mathcal{A}(\mathbb{C})$, we have, in particular,

$$
\left\langle\zeta, r^{-z}\right\rangle=\sum_{j=0}^{N} \sum_{k=0}^{m_{j}}(-1)^{k} c_{j k}(x) \hat{v}_{j k}(\tau) r^{-p_{j}} \log ^{k} r .
$$

Then (2.1.4) takes the form

$$
u(r, x, t)=F_{\tau \rightarrow t}^{1}\left\{[\tau]_{\ell}^{\frac{n+1}{2}} \omega\left(r[\tau]_{\ell}\right)\left\langle\zeta,\left(r[\tau]_{\ell}\right)^{-z}\right\rangle\right\}+u_{\Theta}(r, x, t) .
$$

With $\mathbb{M}$, the stretched spatial manifold in the sense of Section 1.1 of dimension $1+n$, we associate the double manifold $2 \mathbb{M}$ (obtained by gluing two copies of $\mathbb{M}$ together along the common boundary $\partial \mathbb{M})$. Let $L_{\mathrm{cl}}^{\mu}\left(2 \mathbb{M} ; \mathbb{R}_{\tau}\right)_{(\ell)}$ denote the space of all classical (anisotropic) pseudo-differential operators on $2 \mathbb{M}$ of order $\mu$ the local amplitude functions of which anisotropically depend on the parameter $\tau$ of anisotropy $\ell$. More precisely, such amplitude functions can be written as asymptotic expansions $a(\widetilde{x}, \widetilde{\xi}, \tau) \sim \sum_{j=0}^{\infty} a_{(\mu-j)}(\widetilde{x}, \widetilde{\xi}, \tau) \chi(\widetilde{\xi}, \tau)$, where $a_{(\mu-j)}(\widetilde{x}, \widetilde{\xi}, \tau) \in C^{\infty}\left(U \times\left(\mathbb{R}^{n+2} \backslash\{0\}\right)\right)$, satisfy $a_{(\mu-j)}\left(\widetilde{x}, \lambda \widetilde{\xi}, \lambda^{\ell} \tau\right)=\lambda^{\mu-j} a_{(\mu-j)}(\widetilde{x}, \widetilde{\xi}, \tau)$ for all $\lambda \in \mathbb{R}_{+}$, all $\widetilde{x} \in U,(\widetilde{\xi}, \tau) \neq 0, j \in \mathbb{N}$, and $\chi$ is an excision function.

For every $\mu \in \mathbb{R}$ there exists an element $R^{\mu}(\tau) \in L_{\mathrm{cl}}^{\mu}(2 \mathbb{M}, \mathbb{R})_{(\ell)}$ that induces isomorphisms

$$
R^{\mu}(\tau): H^{s}(2 \mathbb{M}) \rightarrow H^{s-\mu}(2 \mathbb{M})
$$

for all $s \in \mathbb{R}$ and all $\tau \in \mathbb{R}$. We then define the anisotropic space $H^{s}(2 \mathbb{M} \times \mathbb{R})_{(\ell)}, s \in \mathbb{R}$, to be the completion of $C_{0}^{\infty}(2 \mathbb{M} \times \mathbb{R})$ with respect to the norm

$$
\left\{\int\left\|R^{s}(\tau) \hat{u}(\tau)\right\|_{L^{2}(2 \mathbb{M})}^{2} d \tau\right\}^{\frac{1}{2}}
$$

Let $\mathrm{r}_{\text {int } \mathbb{M}}$ denote the operator of restriction $\left.u \rightarrow u\right|_{\mathrm{int} \mathbb{M}}$, and set $H^{s}(\mathbb{M} \times \mathbb{R})_{(\ell)}=\mathrm{r}_{\mathrm{int} \mathbb{M}} H^{s}(2 \mathbb{M} \times$ $\mathbb{R})_{(\ell)}$, equipped with the quotient norm from $H^{s}(2 \mathbb{M} \times \mathbb{R})_{(\ell)}$ that identifies elements $u_{1}$ and $u_{2}$ in that space if $r_{i n t} \mathbb{M}\left(u_{1}-u_{2}\right)=0$. Choose a collar neighbourhood $U$ of $\partial \mathbb{M}$ in $\mathbb{M}$ with a splitting of coordinates $\widetilde{x}=(r, x) \in[0,1] \times X$, and let $\omega(r)$ be an arbitrary cut-off function with $\omega(r)=0$ for $r>1-\varepsilon$ for some $0<\varepsilon<1$.

We then define the spaces

$$
\mathcal{W}^{s, \gamma}(\mathbb{M} \times \mathbb{R})_{(\ell)}=[\omega] \mathcal{W}^{s, \gamma}\left(X^{\wedge} \times \mathbb{R}\right)_{(\ell)}+[1-\omega] H^{s}(\mathbb{M} \times \mathbb{R})_{(\ell)}
$$

and

$$
\mathcal{W}_{P}^{s, \gamma}(\mathbb{M} \times \mathbb{R})_{(\ell)}=[\omega] \mathcal{W}_{P}^{s, \gamma}\left(X^{\wedge} \times \mathbb{R}\right)_{(\ell)}+[1-\omega] H^{s}(\mathbb{M} \times \mathbb{R})_{(\ell)} .
$$

It can be easily proved that this is a correct definition, i.e., if $\varphi \in C_{0}^{\infty}\left(\mathbb{R}_{+}\right)$is any function with compact support in $(0,1)$, then $[\varphi] \mathcal{W}^{s, \gamma}(\mathbb{M} \times \mathbb{R})_{(\ell)}=[\varphi] H^{s}(\mathbb{M} \times \mathbb{R})_{(\ell)}$.

\subsection{Continuous edge asymptotics with discrete limit at infinity}

As noted in the beginning, asymptotics of solutions to anisotropic elliptic operators $A u=f$ on $\mathbb{M} \times \mathbb{R}$ with $A$ being of the form (2.1.2) are expected to be like (0.0.1), provided $f$ has asymptotics of analogous structure. We concentrate in this paper on the long-time effect and disregard some details from finite time intervals (these are very interesting, too, cf. Schulze [17], [18] or Schulze and Witt [23] for the isotropic case). In any case the concept of continuous asymptotics is helpful, cf. [11]. 
We employ this under the aspect of a discrete limit at infinity that we introduce in this section.

Let $E$ be a Fréchet space and let $\mathcal{A}(U, E)$ for an open set $U \subset \mathbb{C}$ denote the space of all holomorphic functions in $U$ with values in $E$. Moreover, if $K \subset \subset \mathbb{C}$ is any compact set, $\mathcal{A}^{\prime}(K, E)$ will denote the space of all analytic functionals carried by $K$ with values in $E$ (recall that $\mathcal{A}^{\prime}(K)=\mathcal{A}^{\prime}(K, \mathbb{C})$ is a nuclear Fréchet space in a natural way; then $\left.\mathcal{A}^{\prime}(K, E)=\mathcal{A}^{\prime}(K) \widehat{\otimes}_{\pi} E\right)$. In the present application we set $E=C^{\infty}(X)$, where $X$ is the base of our conical singularity.

Let $V^{I}$ for any $V \subset \mathbb{C}$ denote the smallest closed set containing $V$ such that $z_{0}, z_{1} \in V^{I}$, and $\operatorname{Re} z_{0}=\operatorname{Re} z_{1}$ implies $(1-\lambda) z_{0}+\lambda z_{1} \in V^{I}$ for all $0 \leq \lambda \leq 1$.

Let $V \in \mathbb{C}, V=V^{I}$, and assume that $V \subset\left\{z: \operatorname{Re} z<\frac{n+1}{2}-\gamma\right\}$ for some $\gamma \in \mathbb{R}$ and $V \cap\left\{z: c \leq \operatorname{Re} z \leq c^{\prime}\right\}$ compact for every $c \leq c^{\prime}$. Then, if we fix any $R \geq 0$, the set $K:=V \cap\left\{z: \frac{n+1}{2}-\gamma-R<\operatorname{Re} z\right\}$ is compact, and we have $K=K^{I}$. Choose any (say smooth) curve $C^{2}$ surrounding $K$ clockwise and a function $f \in \mathcal{A}(\mathbb{C} \backslash K, E)$. Then the expression

$$
\zeta: h \rightarrow \frac{1}{2 \pi i} \int_{C} f(z) h(z) d z, \quad h \in \mathcal{A}(\mathbb{C})
$$

defines an element $\zeta \in \mathcal{A}^{\prime}(K, E)$; conversely, every $\zeta \in \mathcal{A}^{\prime}(K, E)$ can be written in this form for a suitable $f \in \mathcal{A}(\mathbb{C} \backslash K, E)$ (the property $K=K^{I}$ is not really necessary, though for several reasons it is convenient to impose it, e.g., to avoid bounded connected components of $\mathbb{C} \backslash K)$.

Example 2.2.1 Let $E=C^{\infty}\left(X, \hat{H}^{s / \ell}(\mathbb{R})\right)$ and $K=\left\{p_{0}, \ldots, p_{N}\right\}$. Then (2.1.5) can be expressed in the form (2.2.1) for

$$
f(z)=\sum_{j=0}^{N} \sum_{k=0}^{m_{j}}(-1)^{k} k ! c_{j k}(x) \hat{v}_{j k}(\tau)\left(z-p_{j}\right)^{-(k+1)} .
$$

For any fixed choice of a cut-off function $\omega(r)$ we set

$$
\mathcal{E}_{K}\left(X^{\wedge}\right)=\left\{\omega\left\langle\zeta, r^{-z}\right\rangle: \zeta \in \mathcal{A}^{\prime}\left(K, C^{\infty}(X)\right)\right\}
$$

here, $\zeta$ is applied to $r^{-z}$ as a holomorphic function in $z$.

The space (2.2.2) is Fréchet by an isomorphism $\mathcal{E}_{K}\left(X^{\wedge}\right) \cong \mathcal{A}^{\prime}\left(K, C^{\infty}(X)\right)$. In fact, consider the Mellin transform $(M u)(z)=\int_{0}^{\infty} r^{z-1} u(r) d r$ (first for $u \in C_{0}^{\infty}\left(\mathbb{R}_{+}, E\right)$, and then extended to more general function and distribution spaces), and form the weighted Mellin transform $\left(M_{\gamma} u\right)(z)=M\left(r^{-\gamma} u\right)(z+\gamma), \gamma \in \mathbb{R}$. Then we have $f(z)=M_{\gamma}\left(\omega\left\langle\zeta, r^{-w}\right\rangle\right) \in$ $\mathcal{A}(\mathbb{C} \backslash K, E)$, and (2.2.1) reproduces $\zeta$. Now $\mathcal{E}_{K}\left(X^{\wedge}\right)$ is a subspace of $\mathcal{K}^{\infty, \gamma}\left(X^{\wedge}\right)$.

Let us define the non-direct sum of Fréchet spaces

$$
\mathcal{E}_{K}\left(X^{\wedge}\right)+\mathcal{K}_{\Theta}^{s, \gamma}\left(X^{\wedge}\right)
$$

for any fixed finite weight interval $\Theta=(\vartheta, 0]$. The space (2.2.3) is independent of the choice of $R$ when $R>-\vartheta$ if the set $V$ is given and $K$ defined by the above-mentioned intersection. Given $u, v \in \mathcal{E}_{K}\left(X^{\wedge}\right)$ we write $u \sim v$ if $u-v \in \mathcal{K}_{\Theta}^{\infty, \gamma}\left(X^{\wedge}\right)$. Then the quotient space $P=\mathcal{E}_{K}\left(X^{\wedge}\right) / \sim$ is called a continuous asymptotic type, and we set $\mathcal{K}_{P}^{s, \gamma}\left(X^{\wedge}\right):=$ $\mathcal{E}_{K}\left(X^{\wedge}\right)+\mathcal{K}_{\Theta}^{s, \gamma}\left(X^{\wedge}\right)$. To define spaces with continuous asymptotics for an infinite weight interval we proceed as follows: First observe that in the construction before we may set $K=V \cap\left\{z: \frac{n+1}{2}-\gamma+\vartheta-1<\operatorname{Re} z\right\}$ without changing that space. Starting from the set 
$V$, to every finite $\vartheta:=-j, j \in \mathbb{N}$, we then get a continuous asymptotic type $P_{j}$ (namely $\left.\mathcal{E}_{K_{j}}\left(X^{\wedge}\right) / \sim\right)$, and we can pass to the projective limit

$$
\mathcal{K}_{P}^{s, \gamma}\left(X^{\wedge}\right):=\lim _{j} \mathcal{K}_{P_{j}}^{s, \gamma}\left(X^{\wedge}\right),
$$

the subspace of $\mathcal{K}^{s, \gamma}\left(X^{\wedge}\right)$ with continuous asymptotics of type $P$, associated with $V$ and the infinite weight interval $(-\infty, 0]$. In other words, by suitable projective limits we can pass from finite to infinite weight intervals $\Theta$.

We can adopt notation from the preceding section for the case of continuous asymptotics and get a corresponding space $\mathcal{W}_{P}^{s, \gamma}(\mathbb{M} \times \mathbb{R})_{(\ell)}$ for each continuous asymptotic type $P$. In this definition $P$ is formally constant with respect to $t$, insofar the carrier set $V$ is independent of $t$. However, we can single out subspaces with a specific control of dependence of "coefficients" of asymptotics on $t$ that allows us to consider variable $V$ and to describe a limit for $t \rightarrow \infty$.

Let us choose any smooth function $\tau \rightarrow[\tau]_{\ell}$ that has the property $[\tau]_{\ell}>0$ for all $\tau \in \mathbb{R}$ and $[\tau]_{\ell}=|\tau|^{\frac{1}{\ell}}$ for $|\tau| \geq c$ for some $c>0$.

We want to employ the following analogue of Proposition 3.1.32 from [19].

Proposition 2.2.2 Let $P$ be a continuous asymptotic type associated with a carrier set $V \subset\left\{z \in \mathbb{C}: \operatorname{Re} z<\frac{n+1}{2}-\gamma\right\}$ that has the above-mentioned properties. Then, every $u(r, x, t) \in \mathcal{W}_{P}^{s, \gamma}\left(X^{\wedge} \times \mathbb{R}\right)_{(\ell)}$ can be written in the form

$$
u(r, x, t)=F_{\tau \rightarrow t}^{-1}\left\{[\tau]_{\ell}^{\frac{n+1}{2}} \omega\left(r[\tau]_{\ell}\right)\left\langle\zeta_{\Theta},\left(r[\tau]_{\ell}\right)^{-z}\right\rangle\right\}+u_{\Theta}(r, x, t)
$$

for every finite $\Theta=(\vartheta, 0], \vartheta<0$, for a certain element

$$
\zeta_{\Theta} \in \mathcal{A}^{\prime}\left(K_{\Theta}, C^{\infty}\left(X, \hat{H}^{s / \ell}(\mathbb{R})\right),\right.
$$

where $\hat{H}^{s / \ell}(\mathbb{R})=F_{t \rightarrow \tau} H^{s / \ell}(\mathbb{R}), K_{\Theta}=V \cap\left\{z: \operatorname{Re} z \geq \frac{n+1}{2}-\gamma+\vartheta-1\right\}$ and $u_{\Theta}(r, x, t) \in$ $\mathcal{W}_{\Theta}^{s, \gamma}\left(X^{\wedge} \times \mathbb{R}\right)_{(\ell)}$.

We can write

$$
\mathcal{A}^{\prime}\left(K_{\Theta}, C^{\infty}\left(X, \hat{H}^{s / \ell}(\mathbb{R})\right)=\mathcal{A}^{\prime}\left(K_{\Theta}, C^{\infty}(X)\right) \widehat{\otimes}_{\pi} \hat{H}^{s / \ell}(\mathbb{R})\right.
$$

and thus represent elements $\zeta_{\Theta}$ in that space as convergent sums

$$
\zeta_{\Theta}=\sum_{j=0}^{\infty} \lambda_{j} \zeta_{\Theta, j} \hat{v}_{j},
$$

$\lambda_{j} \in \mathbb{C}$, such that $\sum_{j=0}^{\infty}\left|\lambda_{j}\right|<\infty$ and sequences $\zeta_{\Theta, j} \in \mathcal{A}^{\prime}\left(K_{\Theta}, C^{\infty}(X)\right), v_{j} \in H^{s / \ell}(\mathbb{R})$, tending to zero for $j \rightarrow \infty$ in the respective spaces. Inserting (2.2.6) into the corresponding expression in (2.2.4) we get a convergent sum. Then, similarly to the representation of singular functions for discrete asymptotics in (2.1.4), also in the continuous case the singular functions are expressed in terms of elements

$$
F_{\tau \rightarrow t}^{-1}\left\{[\tau]_{\ell}^{\frac{n+1}{2}} \omega\left(r[\tau]_{\ell}\right)\left\langle\zeta_{\Theta},\left(r[\tau]_{\ell}\right)^{-z}\right\rangle \hat{v}(\tau)\right\}
$$

for suitable $\zeta_{\Theta} \in \mathcal{A}^{\prime}\left(K_{\Theta}, C^{\infty}(X)\right), v \in H^{s / \ell}(\mathbb{R})$. Recall that also the singular functions on the right hand side of $(2.1 .4)$ can be written in the form $(2.2 .7)$, where $\zeta_{\Theta}$ is represented via (2.2.1) by a $C^{\infty}(X)$-valued meromorphic function $f(z)$ with poles $p_{j}$ of multiplicities $m_{j}+1$ and Laurent coefficients at $\left(z-p_{j}\right)^{-(k+1)}$ belonging to $L_{j}, 0 \leq k \leq m_{j}$. In other words, both in the discrete and continuous case we may look at the expressions (2.2.7). 
Remark 2.2.3 The space $\mathcal{W}_{P}^{s, \gamma}\left(X^{\wedge} \times \mathbb{R}\right)_{(\ell)}$ for a continuous asymptotic type $P$ (of carrier $V)$ remains unchanged when we admit the analytic functionals $\zeta$ in (2.2.7) to be smooth functions in $t \in \mathbb{R}$ up to $t= \pm \infty$, more precisely, $\zeta \in C^{\infty}\left(\mathbb{R}, \mathcal{A}^{\prime}\left(K_{\Theta}, C^{\infty}(X)\right)\right.$, and $\zeta( \pm \log \widetilde{t}) \in C^{\infty}\left(\overline{\mathbb{R}}_{+}, \mathcal{A}^{\prime}\left(K_{\Theta}, C^{\infty}(X)\right)\right.$. A corresponding local result (with respect to the edge variable) in the isotropic set-up is proved in [19], Proposition 3.1.35. The corresponding global (with respect to $t \in \mathbb{R}$ ) result can be obtained by interpreting expression (2.2.7) as $\mathrm{Op}(k) v$ for a suitable operator-valued symbol $k(t, \tau)$. The latter aspect is of crucial importance, so we formulate this separately in Section 2.3 below.

Remark 2.2.4 Singular functions in the frame of continuous asymptotics with $\zeta(t) \in$ $C^{\infty}\left(\mathbb{R}, \mathcal{A}^{\prime}\left(K_{\Theta}, C^{\infty}\left(X, \hat{H}^{s / \ell}(\mathbb{R})\right)\right)\right.$ are able to express $t$-dependent discrete asymptotics, similarly to the scenario discussed in Schulze [12], [17], [18]. It suffices to insert functions $\zeta(t)$ that are $t$-wise discrete and of finite order, given by $t$-dependent $C^{\infty}\left(X, \hat{H}^{s / \ell}(\mathbb{R})\right)$-valued meromorphic functions $f(t, z)$ with poles $p_{j}(t)$ and multiplicities $m_{j}(t)+1$ depending on $t$, cf. Example 2.2.1.

Definition 2.2.5 An element $\zeta(t) \in C^{\infty}\left(\mathbb{R}, \mathcal{A}^{\prime}\left(K_{\Theta}, C^{\infty}(X)\right)\right), \Theta=(\vartheta, 0]$ finite, is called smooth up to $t=\infty$ if $\widetilde{\zeta}(\widetilde{t}):=\zeta(-\log \widetilde{t})$ has the property $\widetilde{\zeta}(\widetilde{t}) \in C^{\infty}\left(\overline{\mathbb{R}}_{+}, \mathcal{A}^{\prime}\left(K_{\Theta}, C^{\infty}(X)\right)\right.$. The function $\zeta(t)$ is said to have a discrete limit at infinity if $\widetilde{\zeta}(0)$ is discrete, i.e., there is a discrete asymptotic type $P=\left\{\left(p_{j}, m_{j}, L_{j}\right)\right\}$ such that $\pi_{\mathbb{C}} P \subset\left\{z: \frac{n+1}{2}-\gamma+\vartheta<\operatorname{Re} z<\right.$ $\left.\frac{n+1}{2}-\gamma\right\}$ and $\langle\widetilde{\zeta}(0), h\rangle=\left.\sum_{j} \sum_{k=0}^{m_{j}} c_{j k} \frac{d^{k}}{d z^{k}} h(z)\right|_{z=p_{j}}, h \in \mathcal{A}(\mathbb{C})$, with coefficients $c_{j k} \in L_{j}$, $0 \leq k \leq m_{j}$, for all $j$.

\subsection{Calculus with operator-valued symbols}

Let us now turn to (anisotropic) operator-valued symbols that are involved in several variants in our final result on long-time asymptotics.

Definition 2.3.1 Let $\left(E,\left\{\kappa_{\lambda}\right\}_{\lambda \in \mathbb{R}_{+}}\right)$and $\left(\widetilde{E},\left\{\widetilde{\kappa}_{\lambda}\right\}_{\lambda \in \mathbb{R}_{+}}\right)$be Hilbert spaces with group actions. Then $S^{\mu}(\Omega \times \mathbb{R} ; E, \widetilde{E})_{(\ell)}$ for any open $\Omega \subset \mathbb{R}$ is defined to be the space of all $a(t, \tau) \in C^{\infty}(\Omega \times \mathbb{R}, \mathcal{L}(E, \widetilde{E}))$ such that

$$
\left\|\widetilde{\kappa}_{\langle\tau\rangle_{\ell}}^{-1}\left\{D_{t}^{k} D_{\tau}^{j} a(t, \tau)\right\} \kappa_{\langle\tau\rangle_{\ell}}\right\|_{\mathcal{L}(E, \widetilde{E})} \leq c\langle\tau\rangle_{\ell}^{\mu-j}
$$

for all $k, j \in \mathbb{N}$ and all $t \in K$ for arbitrary $K \subset \subset \Omega, \tau \in \mathbb{R}$, with constants $c=c(k, j, K)>0$.

More generally, we shall employ symbol classes $S^{\mu}(\Omega \times \mathbb{R} ; E, \widetilde{E})_{(\ell)}$ when $E$ or $\widetilde{E}$ are Fréchet spaces with group actions. For $E=\mathbb{C}^{N}$ we always set $\kappa_{\lambda}=\operatorname{id}_{E}, \lambda \in \mathbb{R}_{+}$. There is also a notion of (anisotropic) classical symbols in terms of asymptotic expansions into functions $a_{(\mu-j)}(t, \tau) \in C^{\infty}(\Omega \times(\mathbb{R} \backslash\{0\}), \mathcal{L}(E, \widetilde{E})), j \in \mathbb{N}$, such that

$$
a_{(\mu-j)}\left(t, \lambda^{\ell} \tau\right)=\lambda^{\mu-j} \widetilde{\kappa}_{\lambda} a_{(\mu-j)}(t, \tau) \kappa_{\lambda}^{-1}
$$

for all $(t, \tau) \in \Omega \times(\mathbb{R} \backslash 0), \lambda \in \mathbb{R}_{+}$. Let $S_{\mathrm{cl}}^{\mu}(\Omega \times \mathbb{R} ; E, \widetilde{E})_{(\ell)}$ denote the corresponding subspace of classical symbols.

Given an element $a(t, \tau) \in S^{\mu}(\Omega \times \mathbb{R} ; E, \widetilde{E})_{(\ell)}$ we set $\mathrm{Op}(a) u(t)=\iint e^{i\left(t-t^{\prime}\right) \tau} a(t, \tau) u\left(t^{\prime}\right)$ $d t^{\prime} d \tau, d \tau=(2 \pi)^{-1} d \tau$. Then $\mathrm{Op}(a): C_{0}^{\infty}(\Omega, E) \rightarrow C^{\infty}(\Omega, \widetilde{E})$ is a continuous operator. In particular, let us set $\Omega=\mathbb{R}$, and let $S_{\mathrm{b}(\mathrm{cl})}^{\mu}(\mathbb{R} \times \mathbb{R} ; E, \widetilde{E})_{(\ell)}$ denote the subspace of all $S_{(\mathrm{cl})}^{\mu}(\mathbb{R} \times \mathbb{R} ; E, \widetilde{E})_{(\ell)}$ such that the symbol estimates of $(2.3 .1)$ hold uniformly for all $(t, \tau) \in$ $\mathbb{R} \times \mathbb{R}$ (subscript (cl) means that we talk about general or classical spaces). In particular, $S_{(\mathrm{cl})}^{\mu}(\mathbb{R} ; E, \widetilde{E})_{(\ell)}$, the subspace of all $a(\tau) \in S_{(\mathrm{cl})}^{\mu}(\mathbb{R} \times \mathbb{R} ; E, \widetilde{E})_{(\ell)}$ that are independent of $t$, is contained in $S_{\mathrm{b}(\mathrm{cl})}^{\mu}(\mathbb{R} \times \mathbb{R} ; E, \widetilde{E})_{(\ell)}$. 
Theorem 2.3.2 Let $a(t, \tau) \in S_{\mathrm{b}}^{\mu}(\mathbb{R} \times \mathbb{R} ; E, \widetilde{E})_{(\ell)}$; then $\mathrm{Op}(a)$ extends to a continuous operator

$$
\mathrm{Op}(a): \mathcal{W}^{s}(\mathbb{R}, E)_{(\ell)} \rightarrow \mathcal{W}^{s-\mu}(\mathbb{R}, \widetilde{E})_{(\ell)}
$$

for every $s \in \mathbb{R}$.

For a proof, cf. Seiler [25]. Theorem 3.14, combined with Corollary 3.16 to reduce orders.

The spaces $S_{\mathrm{b}(\mathrm{cl})}^{\mu}(\mathbb{R} \times \mathbb{R} ; E, \widetilde{E})_{(\ell)}$ contain several interesting subclasses, namely those with exit behaviour of order zero as well as with exponential stabilisation for $|t| \rightarrow \infty$. More precisely, we have $S_{\mathrm{e}}^{\mu}(\mathbb{R} \times \mathbb{R} ; E, \widetilde{E})_{(\ell)}$, defined by the system of symbol estimates $\left\|\widetilde{\kappa}_{\langle\tau\rangle_{\ell}}^{-1}\left\{D_{t}^{k} D_{\tau}^{j} a(t, \tau)\right\} \kappa_{\langle\tau\rangle_{\ell}}\right\|_{\mathcal{L}(E, \widetilde{E})} \leq c\langle t\rangle^{-k}\langle\tau\rangle_{\ell}^{\mu-j}$ for all $k, j \in \mathbb{N}$ and all $(t, \tau) \in \mathbb{R} \times \mathbb{R}$, $c=c(k, j)>0$, and the space $S_{c}^{\mu}(\mathbb{R} \times \mathbb{R} ; E, \widetilde{E})_{(\ell)}$, defined to be the set of all $a(t, \tau) \in$ $S_{\mathrm{e}}^{\mu}(\mathbb{R} \times \mathbb{R} ; E, \widetilde{E})_{(\ell)}$ such that $\omega(\widetilde{t}) a( \pm \log \widetilde{t}, \tau) \in S^{\mu}\left(\overline{\mathbb{R}}_{+, \widetilde{t}} \times \mathbb{R} ; E, \widetilde{E}\right)_{(\ell)}$ for any cut-off function $\omega$, where the symbol estimates for the latter spaces are required uniformly up to $\widetilde{t}=0$. Similarly, we define the subspaces with subscript cl. We then have altogether

$$
\begin{aligned}
& S_{(\mathrm{cl})}^{\mu}(\mathbb{R} ; E, \widetilde{E})_{(\ell)} \subset S_{\mathrm{c}(\mathrm{cl})}^{\mu}(\mathbb{R} \times \mathbb{R} ; E, \widetilde{E})_{(\ell)} \\
& \subset S_{\mathrm{e}(\mathrm{cl})}^{\mu}(\mathbb{R} \times \mathbb{R} ; E, \widetilde{E})_{(\ell)} \subset S_{\mathrm{b}(\mathrm{cl})}^{\mu}(\mathbb{R} \times \mathbb{R} ; E, \widetilde{E})_{(\ell)} .
\end{aligned}
$$

Analogous considerations make sense when $E$ or $\widetilde{E}$ are Fréchet spaces with group actions in the above sense. In particular, Theorem 2.3.2 remains true also in this case.

Proposition 2.3.3 Let $\zeta_{\Theta} \in \mathcal{A}^{\prime}\left(K_{\Theta}, C^{\infty}(X)\right.$ ) (in the notation of Proposition 2.2.2) and set $a(\tau)=[\tau]_{\ell}^{\frac{n+1}{2}} \omega\left(r[\tau]_{\ell}\right)\left\langle\zeta_{\Theta},\left(r[\tau]_{\ell}\right)^{-z}\right\rangle$, regarded as a map $a(\tau): \mathbb{C} \rightarrow \mathcal{K}^{\infty, \gamma}\left(X^{\wedge}\right)$. Then we have $a(\tau) \in S_{\mathrm{cl}}^{0}\left(\mathbb{R} ; \mathbb{C}, \mathcal{K}^{\infty, \gamma}\left(X^{\wedge}\right)\right)_{(\ell)}$, and hence

$$
\mathrm{Op}(a): H^{s / \ell}(\mathbb{R}) \rightarrow \mathcal{W}^{s}\left(X^{\wedge} \times \mathbb{R}\right)_{(\ell)}
$$

is continuous for every $s \in \mathbb{R}$.

Proof. Writing $E=\mathbb{C}, \widetilde{E}=\mathcal{K}^{\infty, \gamma}\left(X^{\wedge}\right)$, where $E$ is endowed with $\operatorname{id}_{E}$ for all $\lambda \in \mathbb{R}_{+}$ and $\widetilde{E}$ with the group action $\left\{\kappa_{\lambda}\right\}_{\lambda \in \mathbb{R}_{+}}$induced by that on $\mathcal{K}^{s, \gamma}\left(X^{\wedge}\right)$ for any $s$, we have $a(\tau) \in C^{\infty}(\mathbb{R}, \mathcal{L}(E, \widetilde{E}))$ and $a\left(\lambda^{\ell} \tau\right)=\kappa_{\lambda} a(\tau)$ for all $\lambda \geq 1,|\tau| \geq c$ for some $c>0$. This yields $a(\tau) \in S_{\mathrm{cl}}^{0}(\mathbb{R} ; E, \widetilde{E})_{(\ell)}$. The continuity $(2.3 .2)$ is a simple special case of Theorem 2.3.2.

Remark 2.3.4 The expressions (2.2.7) of the singular functions of the edge asymptotics are nothing else than $\mathrm{Op}(a)$ v for $v \in H^{s / \ell}(\mathbb{R})$.

\section{Corner asymptotics at infinity}

Asymptotics of solutions will be studied in combination with anisotropic elliptic regularity in weighted Sobolev spaces with iterated edge-corner asymptotics for $t \rightarrow \infty$. We state the results under natural weight conditions and trace and potential data along the time-edge.

\subsection{The structure of singular functions}

Using the material of Section 2.1 we can introduce weighted Sobolev spaces $\mathcal{H}^{s, \gamma}(\mathbb{M}):=\{u \in$ $H_{\text {loc }}^{s}($ int $\left.\mathbb{M}): \omega u \in \mathcal{K}^{s, \gamma}\left(X^{\wedge}\right)\right\}, s, \gamma \in \mathbb{R}$, where a collar neighbourhood of $\partial \mathbb{M}$ is identified with $[0,1) \times X$, and $\omega$ is a cut-off function vanishing for $r>1-\varepsilon$ for some $0<\varepsilon<1$. 
Moreover, for every (discrete or continuous) asymptotic type $P$ we set $\mathcal{H}_{P}^{s, \gamma}(\mathbb{M})=\{u \in$ $\left.\mathcal{H}^{s, \gamma}(\mathbb{M}): \omega u \in \mathcal{K}_{P}^{s, \gamma}\left(X^{\wedge}\right)\right\}$. This is a Fréchet space in a natural way. In particular, we consider the spaces $\mathcal{H}_{P}^{\infty, \gamma}(\mathbb{M})=\bigcap_{s \in \mathbb{R}} \mathcal{H}_{P}^{s, \gamma}(\mathbb{M})$.

Given weight data $(\delta, \Xi)$, where $\delta \in \mathbb{R}$ is an exponential weight for $t=\infty$ and $\Xi=(\xi, 0]$ a weight interval that is first assumed to be finite, we call a sequence

$$
Q=\left\{\left(q_{\iota}, n_{\iota}, H_{\iota}\right)\right\}_{\iota=0, \ldots, N},
$$

for $N=N(Q)<\infty$ a discrete corner asymptotic type associated with $(\delta, \Xi)$ if $q_{\iota} \in \mathbb{C}$, $\frac{n+2}{2}-\delta+\xi<\operatorname{Re} q_{\iota}<\frac{n+2}{2}-\delta, n_{\iota} \in \mathbb{N}$, and $H_{\iota}$ is a finite-dimensional subspace of $\mathcal{H}_{P}^{-\infty, \gamma}(\mathbb{M})$ for a certain asymptotic type $P, 0 \leq \iota \leq N$. If $\Xi$ is infinite we admit $N=\infty$ and require in that case $\operatorname{Re} q_{\iota} \rightarrow-\infty$ as $\iota \rightarrow \infty$, where $P$ is independent of $\iota$. We call $P$ a cone asymptotic type belonging to $Q$.

If $P$ is any cone asymptotic type and $\Xi=(\xi, 0]$ a finite weight interval, we set

$$
\begin{aligned}
& \mathcal{W}_{P, \Xi}^{s, \gamma ; \delta}(\mathbb{M} \times \mathbb{R})_{(\ell)} \\
& \quad=\bigcap_{\varepsilon>0} \chi(t) e^{-(\delta-\xi-\varepsilon) t} \mathcal{W}_{P}^{s, \gamma}(\mathbb{M} \times \mathbb{R})_{(\ell)}+(1-\chi(t)) e^{-\delta t} \mathcal{W}_{P}^{s, \gamma}(\mathbb{M} \times \mathbb{R})_{(\ell)},
\end{aligned}
$$

where $\chi(t) \in C^{\infty}(\mathbb{R})$ is any function with $\chi(t)=0$ for $t<t_{0}$ and $\chi(t)=1$ for $t>t_{1}$ for certain $t_{0}<t_{1}$. The space (3.1.1) is Fréchet in a natural way. For $\Xi=(-\infty, 0]$ we define $\mathcal{W}_{P, \Xi}^{s, \gamma ; \delta}(\mathbb{M} \times \mathbb{R})_{(\ell)}$ to be the projective limit over all spaces $\mathcal{W}_{P,(\xi, 0]}^{s, \gamma ; \delta}(\mathbb{M} \times \mathbb{R}), \xi<0$.

Now if $Q$ is a corner asymptotic type to weight data $(\delta, \Xi), \Xi$ finite, and $P$ an associated cone asymptotic type to weight data $(\gamma, \Theta)$, we set

$$
\begin{aligned}
\mathcal{W}_{P, Q}^{s, \gamma ; \delta}(\mathbb{M} \times \mathbb{R})_{(\ell)}=\{u(\widetilde{x}, t) \in & e^{-\delta t} \mathcal{W}_{P}^{s, \gamma}(\mathbb{M} \times \mathbb{R})_{(\ell)}: \\
& \left.u(\widetilde{x}, t)-\chi(t) \sum_{\iota=0}^{N} \sum_{\kappa=0}^{n_{\iota}} \alpha_{\iota \kappa}(\widetilde{x}) e^{q_{\iota} t} t^{\kappa} \in \mathcal{W}_{P, \Xi}^{s, \gamma ; \delta}(\mathbb{M} \times \mathbb{R})_{(\ell)}\right\},
\end{aligned}
$$

where $\alpha_{\iota \kappa}(\widetilde{x})$ are coefficients in $H_{\iota}, 0 \leq \kappa \leq n_{j}$ (uniquely determined by $u$ ), $N=N(Q)$. For $\Xi$ infinite we define the space $\mathcal{W}_{P, Q}^{s, \gamma ; \delta}(\mathbb{M} \times \mathbb{R})_{(\ell)}$ by a corresponding projective limit over finite weight intervals.

The spaces $\mathcal{W}_{P, Q}^{s, \gamma ; \delta}(\mathbb{M} \times \mathbb{R})_{(\ell)}$ define the functional analytic structure of long-time asymptotics of solutions to (anisotropic) elliptic equations $A u=f$ when the right hand sides $f$ belong to a space of analogous nature, namely $\mathcal{W}_{\widetilde{P}, \widetilde{Q}}^{s-\mu, \gamma-\mu ; \delta}(\mathbb{M} \times \mathbb{R})_{(\ell)}$ for certain $\widetilde{P}, \widetilde{Q}$. However, this is only true under some additional control of $u$, connected with the fact that the time-edge behaves like an inner boundary that is responsible for boundary conditions, referring to a weight for $r \rightarrow 0$. Moreover, the corner singularity $t=\infty$ requires some growth condition for $t \rightarrow \infty$. Both aspects are governed by certain (operator-valued) symbols, the so-called edge symbol $\sigma_{\wedge}$ and the corner conormal symbol $\sigma_{\mathrm{c}}$ to be defined below.

\subsection{Operators with trace and potential conditions}

Set $\mathcal{W}^{s, \gamma ; \delta}(\mathbb{M} \times \mathbb{R})_{(\ell)}:=e^{-\delta t} \mathcal{W}^{s, \gamma}(\mathbb{M} \times \mathbb{R})_{(\ell)}, s, \gamma, \delta \in \mathbb{R}$. The given differential operator $A$ induces continuous maps $A: \mathcal{W}^{s, \gamma ; \delta}(\mathbb{M} \times \mathbb{R})_{(\ell)} \rightarrow \mathcal{W}^{s-\mu, \gamma-\mu ; \delta}(\mathbb{M} \times \mathbb{R})_{(\ell)}$ for every $s, \gamma, \delta \in \mathbb{R}$. This is a consequence of the growth conditions on the coefficients and of Theorem 2.3.2 (concerning a neighbourhood of $\partial \mathbb{M} \times \mathbb{R}$ ) as well as of known continuity properties in Sobolev spaces far from $\partial \mathbb{M} \times \mathbb{R}$. Let $H^{s / \ell ; \delta}\left(Y, \mathbb{C}^{N}\right):=e^{-\delta t} H^{s / \ell}(\mathbb{R}) \otimes \mathbb{C}^{N}$ with $H^{\nu}(\mathbb{R})$ being the 
standard Sobolev space on $\mathbb{R}$ of smoothness $\nu$. With $A$ we can connect a block matrix operator

$$
\mathcal{A}=\left(\begin{array}{cc}
A & K \\
T & Q
\end{array}\right): \begin{gathered}
\mathcal{W}^{s, \gamma ; \delta}(\mathbb{M} \times \mathbb{R})_{(\ell)} \\
H^{s / \ell ; \delta}\left(Y, \mathbb{C}^{N_{-}}\right)
\end{gathered} \rightarrow \underset{H^{(s-\mu) / \ell ; \delta}\left(Y, \mathbb{C}^{N_{+}}\right)}{\mathcal{W}^{s-\mu, \gamma-\mu ; \delta}(\mathbb{M} \times \mathbb{R})_{(\ell)}}
$$

that is continuous for all $s \in \mathbb{R}$. Here, $Y$ is the time-edge, (i.e., a disjoint union of copies of $\mathbb{R}$ when $M$ has a finite number of conical points). Recall that for simplicity we have assumed $Y=\mathbb{R}$ (the general case is completely analogous).

The meaning of the entries in (3.2.1) is the same as in the pseudo-differential calculus on a manifold with edge singularities: $T$ is a vector of trace conditions, $K$ a vector of potential conditions, and $Q$ is a matrix of classical pseudo-differential operators on $Y$, cf. [19], Section 3.4.4. Recall that $T$ and $K$ are (modulo smoothing operators) of the classes Op $S_{\mathrm{c}, \mathrm{cl}}^{\mu}\left(\mathbb{R} \times \mathbb{R} ; E, \mathbb{C}^{N_{+}}\right)_{(\ell)}$ and Op $S_{\mathrm{c}, \mathrm{cl}}^{\mu}\left(\mathbb{R} \times \mathbb{R} ; \mathbb{C}^{N_{-}}, \widetilde{E}\right)_{(\ell)}$, respectively, where $E=\mathcal{K}^{s, \gamma}\left(X^{\wedge}\right)$ and $\widetilde{E}=\mathcal{K}^{s-\mu, \gamma-\mu}\left(X^{\wedge}\right)$ for arbitrary $s \in \mathbb{R}$. The symbols have, in fact, more specific properties; for instance, we may replace $\widetilde{E}$ by

$$
\mathcal{S}_{Q}^{\gamma-\mu}\left(X^{\wedge}\right)=\bigcap_{k \in \mathbb{N}}\langle r\rangle^{-k} \mathcal{K}_{Q}^{\infty, \gamma-\mu}\left(X^{\wedge}\right)
$$

for some asymptotic type $Q$ with respect to $r \rightarrow 0$, and trace operators $T$ can be characterised by duality in terms of potential operators of a similar structure, cf. [19]. The operators (3.2.1) can be regarded as elements of a (graded) algebra of block matrix operators (where compositions are defined, when the numbers of rows and columns of the factors fit together), cf. [14] or [19]. Let $\mathcal{C}^{\mu}\left(\mathbb{M} \times \mathbb{R}, \boldsymbol{g} ; N_{-}, N_{+}\right)_{(\ell)}$ for $\boldsymbol{g}=(\gamma, \gamma-\mu, \Theta ; \delta, \Xi)$ denote the subset of all elements of order $\mu$, acting like (3.2.1), where the weight intervals $\Theta=(-(k+1), 0]$, $k \in \mathbb{N}$, (with respect to power weights for $r \rightarrow 0$ ) and $\Xi=(-(\nu+1), 0]$ (with respect to exponential weights for $t \rightarrow \infty$ ) are fixed. From now on we freely use the tools of that algebra, more precisely, an anisotropic variant, according to our anisotropy $\ell$, combined with a modification for $t \rightarrow \pm \infty$ that consists of a corner pseudo-differential calculus in $\widetilde{t}=e^{-t}$ for $\widetilde{t} \rightarrow 0$ in the sense of the theory of [20] and of an analogous calculus in $\widetilde{\widetilde{t}}=e^{t}$ for $\widetilde{\widetilde{t}} \rightarrow 0$ in a version without asymptotics, also in anisotropic form.

To give more details we first introduce the smoothing operators of the theory. Parallel to the wedge spaces with asymptotics in $t$-direction we can also consider spaces $H_{R}^{\nu ; \delta}\left(Y, \mathbb{C}^{N}\right)=$ $H_{R}^{\nu ; \delta}(\mathbb{R}) \otimes \mathbb{C}^{N}$, where $R=\left\{\left(r_{j}, h_{j}\right)\right\}$ are (scalar) asymptotic types, similarly to those for conical singularities, associated with weight data $(\delta, \Xi)$. The simplest way to define these spaces is to set $u \in H_{R}^{\nu, \delta}(\mathbb{R}) \Leftrightarrow\left\{u \in H_{\text {loc }}^{\nu}(\mathbb{R}): \omega\left(\widetilde{t} \tilde{t}^{-\frac{1}{2}} u(-\log \widetilde{t}) \in \mathcal{K}_{R}^{s, \gamma}\left(\mathbb{R}_{+}\right), \psi(t) u \in H^{\nu ; \delta}\right\}\right.$ for any cut-off function $\omega(\widetilde{t}), \widetilde{t} \in \mathbb{R}_{+}$, and a function $\psi \in C^{\infty}(\mathbb{R})$ such that $\psi(t)=1$ for $t<t_{0}, \psi(t)=0$ for $t>t_{1}$ for certain $t_{0}<t_{1}$. Let $\mathcal{C}^{-\infty}\left(\mathbb{M} \times \mathbb{R}, \boldsymbol{g} ; N_{-}, N_{+}\right)$defined to be the space of all continuous operators (3.2.1) that induce continuous operators

$$
\mathcal{C}: \begin{gathered}
\mathcal{W}^{s, \gamma ; \delta}(\mathbb{M} \times \mathbb{R})_{(\ell)} \\
H^{s / \ell ; \delta}\left(Y, \mathbb{C}^{N_{-}}\right)
\end{gathered} \rightarrow \begin{gathered}
\mathcal{W}_{P, Q}^{\infty, \gamma-\mu ; \delta}(\mathbb{M} \times \mathbb{R}) \\
H_{R}^{\infty ; \delta}\left(Y, \mathbb{C}^{N_{+}}\right)
\end{gathered}
$$

for all $s \in \mathbb{R}$, with certain asymptotic types $(P, Q ; R)$ and such that the formal adjoint $\mathcal{C}^{*}$ has analogous mapping properties $\left(\mathcal{C}^{*}\right.$ is taken with respect to the scalar products of the spaces where smoothness and weights are equal to zero). In addition, the involved asymptotic types are assumed to have discrete limits for $t \rightarrow \infty$.

The lower right corner $Q$ of a block matrix operator $\mathcal{A} \in \mathcal{C}^{\mu}\left(\mathbb{M} \times \mathbb{R}, \boldsymbol{g} ; N_{-}, N_{+}\right)_{(\ell)}$ is an $N_{+} \times N_{-}$matrix of elements $Q_{i j} \in L_{\mathrm{cl}}^{\mu}(\mathbb{R})_{(\ell)}\left(=L_{\mathrm{cl}}^{\mu / \ell}(\mathbb{R})\right)$ such that for the diffeomorphisms 
$\chi_{ \pm}: \mathbb{R} \rightarrow \mathbb{R}_{+}, \chi_{ \pm}: t \rightarrow e^{\mp t}$, and cut-off functions $\omega, \widetilde{\omega}$ on $\mathbb{R}_{+}$the operators $\omega\left\{\left(\chi_{ \pm}\right)_{*} Q_{i j}\right\} \widetilde{\omega}$ belong to the cone algebra on $\mathbb{R}_{+}$with discrete asymptotics for the + sign and without asymptotics for the - sign and weight data induced by the choice of $(\delta, \Xi)$, cf. [16], Definition 1.2.1 and [14], Definition 6 in Section 1.2.4 (for $M L_{\mathrm{cl}}^{\mu / \ell}\left(\overline{\mathbb{R}}_{+}\right)$).

Moreover, the space $C^{\mu}(\mathbb{M} \times \mathbb{R}, \boldsymbol{g})_{(\ell)}$ of upper left corners $A$ of operators $\mathcal{A} \in \mathcal{C}^{\mu}(\mathbb{M} \times$ $\left.\mathbb{R}, \boldsymbol{g} ; N_{-}, N_{+}\right)_{(\ell)}$ is a subspace of $L_{\mathrm{cl}}^{\mu}(\text { int } \mathbb{M} \times \mathbb{R})_{(\ell)}$ such that (modulo $C^{-\infty}(\mathbb{M} \times \mathbb{R}, \boldsymbol{g})=$ $\left.\mathcal{C}^{-\infty}(\mathbb{M} \times \mathbb{R}, \boldsymbol{g} ; 0,0)\right)$

(i) for any choice of cut-off functions $\sigma(r), \widetilde{\sigma}(r)$ supported in a collar neighbourhood of $\partial \mathbb{M}$ the operators $\omega\left\{\left(\chi_{ \pm}\right)_{*}(1-\sigma) A(1-\widetilde{\sigma})\right\} \widetilde{\omega}$ belong to the cone algebra on $\mathbb{R}_{+} \times(2 \mathbb{M})$ without asymptotics (i.e., to $\widetilde{t}^{\delta} M L_{\mathrm{cl}}^{\mu / \ell}\left(\overline{\mathbb{R}}_{+} \times(2 \mathbb{M})\right)$ for $\widetilde{t}=\chi_{ \pm}(t)$, cf. [14], page 219),

(ii) $\sigma A \widetilde{\sigma}$ belongs to an anisotropic analogue of the wedge algebra on $X^{\wedge} \times \mathbb{R}$ with continuous asymptotics, cf. [19], Definition 3.4.29, and [3], where the involved operatorvalued symbols are supposed to belong to $S_{\mathrm{c}}^{\mu}(\mathbb{R} \times \mathbb{R} ; E, \widetilde{E})_{(\ell)}$ for $E=\mathcal{K}^{s, \gamma}\left(X^{\wedge}\right)$, $\widetilde{E}=\mathcal{K}^{s-\mu, \gamma-\mu}\left(X^{\wedge}\right), s \in \mathbb{R}$

(iii) let $\chi_{+}: \mathbb{M} \times \mathbb{R} \rightarrow \mathbb{M} \times \mathbb{R}_{+}$denote the map $(\widetilde{x}, t) \rightarrow\left(\widetilde{x}, e^{-t}\right)$ and let $\omega, \widetilde{\omega}$ be cut-off functions in $\tilde{t}=e^{-t} \in \mathbb{R}_{+}$; then $\omega\left\{\left(\chi_{+}\right)_{*} A\right\} \widetilde{\omega}$ belongs to an anisotropic analogue of the corner algebra on $\mathbb{M} \times \mathbb{R}$ in the sense of [15], Definition 3.1.2, in addition, the involved asymptotic types along the one-dimensional edge (both in the Green and the smoothing Mellin symbols) are assumed to have discrete limits for $t \rightarrow \infty$.

Finally, an analogue of condition (iii) is required for the full block matrix $\mathcal{A} \in \mathcal{C}^{\mu}(\mathbb{M} \times$ $\left.\mathbb{R}, \boldsymbol{g} ; N_{-}, N_{+}\right)_{(\ell)}$.

Every $\mathcal{A} \in \mathcal{C}^{\mu}\left(\mathbb{M} \times \mathbb{R}, \boldsymbol{g} ; N_{-}, N_{+}\right)_{(\ell)}$ is continuous in the sense

$$
\mathcal{A}: \begin{aligned}
& \mathcal{W}_{P, Q}^{s, \gamma ; \delta}(\mathbb{M} \times \mathbb{R})_{(\ell)} \\
& H_{R}^{s / \ell ; \delta}\left(Y, \mathbb{C}^{N_{-}}\right)
\end{aligned} \rightarrow \begin{aligned}
& \mathcal{W}_{\widetilde{P}, \widetilde{Q}}^{s-\mu, \gamma-\mu ; \delta}(\mathbb{M} \times \mathbb{R})_{(\ell)} \\
& H_{\widetilde{R}}^{(s-\mu) / \ell ; \delta}\left(Y, \mathbb{C}^{N_{+}}\right)
\end{aligned}
$$

for arbitrary asymptotic types $P, Q$ and $R$ with certain resulting $\widetilde{P}, \widetilde{Q}$, and $\widetilde{R}$ (associated with the corresponding weight data) and all $s \in \mathbb{R}$. If $(P, Q ; R)$ have discrete limits for $t \rightarrow \infty$, then the same is true of $(\widetilde{P}, \widetilde{Q} ; \widetilde{R})$.

The upper left corners of elements $\mathcal{A} \in \mathcal{C}^{\mu}\left(\mathbb{M} \times \mathbb{R}, \boldsymbol{g} ; N_{-}, N_{+}\right)_{(\ell)}$ are classical anisotropic pseudo-differential operators in (int $\mathbb{M}) \times \mathbb{R}$, locally decribed by (scalar) amplitude functions with a homogeneous principal part of order $\mu$,

$$
\sigma_{\psi}(\mathcal{A})(\widetilde{x}, t, \widetilde{\xi}, \tau), \quad(\widetilde{x}, t, \widetilde{\xi}, \tau) \in T^{*}((M \backslash S) \times \mathbb{R}) \backslash 0
$$

In the splitting of covariables $\widetilde{x}$ into $(r, x)$ it takes the form

$$
r^{-\mu} \widetilde{\sigma}_{\psi}(\mathcal{A})\left(r, x, t, r \varrho, \xi, r^{\ell} \tau\right)
$$

where $\left.\widetilde{\sigma}_{\psi}(\mathcal{A}) \underset{\widetilde{t}}{(r}, x, t, \widetilde{\varrho}, \xi, \widetilde{\tau}\right)$ is smooth up to $r=0$ and $\widetilde{\sigma}_{\psi}(\mathcal{A})(r, x, \mp \log \widetilde{t}, \widetilde{\varrho}, \xi, \widetilde{\tau})$ smooth up to $r=0$ and $\widetilde{t}=0$.

Moreover, according to the ideas of the edge pseudo-differential calculus, locally near $\partial \mathbb{M} \times \mathbb{R}$ the operator $\mathcal{A}$ is an anisotropic pseudo-differential operator with an operatorvalued amplitude function of the class $S^{\mu}\left(\mathbb{R}_{t} \times \mathbb{R}_{\tau} ; E, \widetilde{E}\right)_{(\ell)}$ for $E=\mathcal{K}^{s, \gamma}\left(X^{\wedge}\right) \oplus \mathbb{C}^{N_{-}}$, $\widetilde{E}=\mathcal{K}^{s-\mu, \gamma-\mu}\left(X^{\wedge}\right) \oplus \mathbb{C}^{N_{+}}$, for all $s \in \mathbb{R}$, and there is a principal edge symbol.

$$
\sigma_{\wedge}(\mathcal{A})(t, \tau): E \rightarrow \widetilde{E}, \quad(t, \tau) \in \mathbb{R} \times(\mathbb{R} \backslash\{0\}),
$$


where $\sigma_{\wedge}(\mathcal{A})(\mp \log \widetilde{t}, \tau)$ is smooth in $\widetilde{t}$ up to $\widetilde{t}=0$. Finally, there is a principal corner conormal symbol that is responsible for a control of $\mathcal{A}$ for $t \rightarrow \infty$, namely

$$
\sigma_{\mathrm{c}}(\mathcal{A})(w): \mathcal{H}^{s, \gamma}(\mathbb{M}) \oplus \mathbb{C}^{N_{-}} \rightarrow \mathcal{H}^{s-\mu, \gamma-\mu}(\mathbb{M}) \oplus \mathbb{C}^{N_{+}},
$$

$s \in \mathbb{R}$, depending on $w \in \Gamma_{\frac{n+2}{2}-\delta}$ for the chosen weight $\delta \in \mathbb{R}$. Then

$$
\sigma(\mathcal{A})=\left(\sigma_{\psi}(\mathcal{A}), \sigma_{\wedge}(\mathcal{A}), \sigma_{\mathrm{c}}(\mathcal{A})\right)
$$

is called the principal symbol of $\mathcal{A}$. Note that there are various kinds of subordinate symbols, for instance, the principal cone conormal symbol $\sigma_{M}(\mathcal{A})(t, z)$, cf. formula $(1.2 .6)$, or the cone and corner conormal symbols of lower conormal orders that take part in the specific transformation of asymptotic types $(P, Q ; R) \rightarrow(\widetilde{P}, \widetilde{Q} ; \widetilde{R})$ in $(3.2 .3)$.

Theorem 3.2.1 $\mathcal{A} \in \mathcal{C}^{\mu}\left(\mathbb{M} \times \mathbb{R}, \boldsymbol{g} ; N_{0}, N_{+}\right)_{(\ell)}, \mathcal{B} \in \mathcal{C}^{\nu}\left(\mathbb{M} \times \mathbb{R}, \boldsymbol{h} ; N_{-}, N_{0}\right)_{(\ell)}$ for $\boldsymbol{g}=(\gamma-$ $\nu, \gamma-\nu-\mu, \Theta ; \delta, \Xi), \boldsymbol{h}=(\gamma, \gamma-\nu, \Theta ; \delta, \Xi)$ implies $\mathcal{A B} \in \mathcal{C}^{\mu+\nu}\left(\mathbb{M} \times \mathbb{R}, \boldsymbol{g} \circ \boldsymbol{h} ; N_{-}, N_{+}\right)_{(\ell)}$ for $\boldsymbol{g} \circ \boldsymbol{h}=(\gamma, \gamma-\nu-\mu, \Theta ; \delta, \Xi)$, and we have

$$
\sigma(\mathcal{A B})=\sigma(\mathcal{A}) \sigma(\mathcal{B})
$$

with componentwise multiplication.

This result is an anisotropic analogue of [15], Theorem 3.2.9 as far as it concerns $t \leq \infty$, while for $t \rightarrow-\infty$ it corresponding to an anisotropic form of [15], Theorem 1.4.24.

\subsection{Asymptotics and (anisotropic) elliptic regularity}

We now turn to our result on long-time asymptotics of solutions $u \in \mathcal{W}^{-\infty, \gamma ; \delta}(\mathbb{M} \times \mathbb{R})_{(\ell)} \oplus$ $H^{-\infty ; \delta}\left(Y, \mathbb{C}^{N_{-}}\right)$of

$$
\mathcal{A} u=f \in \mathcal{W}_{\widetilde{P}, \widetilde{Q}}^{s-\mu, \gamma-\mu ; \delta}(\mathbb{M} \times \mathbb{R})_{(\ell)} \oplus H_{\widetilde{R}}^{(s-\mu) / \ell ; \delta}\left(Y, \mathbb{C}^{N_{+}}\right)
$$

for given asymptotic types $(\widetilde{P}, \widetilde{Q} ; \widetilde{R})$ where

$$
\mathcal{A} \in \mathcal{C}^{\mu}\left(\mathbb{M} \times \mathbb{R}, \boldsymbol{g} ; N_{-}, N_{+}\right)_{(\ell)}, \quad \boldsymbol{g}=(\gamma, \gamma-\mu, \Theta ; \delta, \Xi) .
$$

The assumption on $u$ may be regarded as a weight control that is imposed on a solution, both for $r \rightarrow 0$ and $t \rightarrow \infty$. Moreover, we talk about functions $u=\left(u_{1}, u_{2}\right)$ where $u_{1}$ is given on $\mathbb{M} \times \mathbb{R}$ and $u_{2}$ on $Y$. This reflects another control of solutions, namely of trace and potential data along the edge $Y$. If we are interested only in $u_{1}$, the control consists of the assumption that the first component $f_{1}$ of $f=\left(f_{1}, f_{2}\right)$ on the right hand side belongs to a certain subspace of $\mathcal{W}_{\widetilde{P}, \tilde{Q}}^{s-\mu, \gamma-\mu ; \delta}(\mathbb{M} \times \mathbb{R})_{(\ell)}$, that is (roughly speaking) characterised as the complement of the image of the potential operator in $\mathcal{A}$, and, in addition, $u_{1}$ takes prescribed edge trace values $f_{2}$.

The upper left corner $A$ of $\mathcal{A}$ may be a differential operator as in Section 1.2, where we assume, in particular, anisotropic ellipticity. Also in this case $\mathcal{A}$ is a block matrix containing extra edge data, required in a way that $\mathcal{A}$ is anisotropic elliptic with respect to $\sigma(\mathcal{A})$, cf. Definition 3.3 .1 below. These additional data are indispensable to get asymptotic results, even for finite $t$ and $r \rightarrow 0$. This belongs to the main aspects of asymptotic phenomena under geometric singularities. The asymptotics themselves, in particular, the singular functions, are expressed in terms of weighted Sobolev spaces with asymptotics, formulated in Section 3.1 above. They show a typical interplay between asymptotic information for $r \rightarrow 0$ and $t \rightarrow \infty$. Asymptotics of solutions will be obtained as an aspect of (anisotropic) elliptic regularity in such weighted Sobolev spaces. 
Definition 3.3.1 An operator (3.3.2) is called (anisotropic) elliptic if

(i) $\sigma_{\psi}(\mathcal{A})(\widetilde{x}, t, \widetilde{\xi}, \tau) \neq 0$ on $T^{*}((M \backslash S) \times \mathbb{R}) \backslash 0$ and $\widetilde{\sigma}_{\psi}(\mathcal{A})(r, x, \mp \log \widetilde{t}, \widetilde{\varrho}, \xi, \widetilde{\tau}) \neq 0$ on $T^{*}([0, \varepsilon) \times X \times[0, \varepsilon)) \backslash 0$ for an $\varepsilon>0$ (where the intervals $[0, \varepsilon)$ refer to $r$ and $\widetilde{t}$, respectively),

(ii) $\sigma_{\wedge}(\mathcal{A})(t, \tau): E \rightarrow \widetilde{E}$ is an isomorphism for all $(t, \tau) \in \mathbb{R} \times(\mathbb{R} \backslash\{0\})$, where $E=$ $\mathcal{K}^{s, \gamma}\left(X^{\wedge}\right) \oplus \mathbb{C}^{N_{-}}, \widetilde{E}=\mathcal{K}^{s-\mu, \gamma-\mu}\left(X^{\wedge}\right) \oplus \mathbb{C}^{N_{+}}, s \in \mathbb{R}$, and $\sigma_{\wedge}(\mathcal{A})(\mp \log \widetilde{t}, \tau): E \rightarrow \widetilde{E}$ is an isomorphism for all $(\widetilde{t}, \tau) \in[0, \varepsilon) \times(\mathbb{R} \backslash\{0\})$ for some $\varepsilon>0$,

(iii) $\sigma_{\mathrm{c}}(\mathcal{A})(w): \mathcal{H}^{s, \gamma}(\mathbb{M}) \oplus \mathbb{C}^{N_{-}} \rightarrow \mathcal{H}^{s-\mu, \gamma-\mu}(\mathbb{M}) \oplus \mathbb{C}^{N_{+}}$is an isomorphism for all $w \in$ $\Gamma_{\frac{n+2}{2}-\delta}, s \in \mathbb{R}$.

Conditions (ii), (iii) refer to some fixed $s=s_{0} \in \mathbb{R}$; they are then valid for all $s \in \mathbb{R}$. Note that condition (ii) is an anisotropic analogue of the Shapiro-Lopatinskij condition for edge singularities.

Remark 3.3.2 A consequence of the assumption on discrete limits for $t \rightarrow \infty$ of the asymptotic types in an elliptic operator $\mathcal{A}$ is that $\sigma_{\mathrm{c}}(\mathcal{A})(w)$ is a meromorphic operator function in $w \in \mathbb{C}$ with values in $\mathcal{L}\left(\mathcal{H}^{s, \gamma}(\mathbb{M}) \oplus \mathbb{C}^{N_{-}}, \mathcal{H}^{s-\mu, \gamma-\mu}(\mathbb{M}) \oplus \mathbb{C}^{N_{+}}\right), s \in \mathbb{R}$, that is invertible outside a discrete set $D \subset \mathbb{C}$ of points such that $D \cap\left\{w: c \leq \operatorname{Re} w \leq c^{\prime}\right\}$ is finite for every $c \leq c^{\prime}$. If $q \in \mathbb{C}$ is a pole of $\sigma_{\mathrm{c}}(\mathcal{A})(w)$, the Laurent coefficients at $(z-q)^{-(k+1)}$, $0 \leq k \leq m(q)$, are of finite rank and Green operators with discrete asymptotics in the sense of the cone algebra on $\mathbb{M}$, augmented by the finite-dimensional entries of our block matrices. The inverse $\sigma_{\mathrm{c}}(\mathcal{A})^{-1}(w)$ is of analogous nature.

Theorem 3.3.3 Let $\mathcal{A} \in \mathcal{C}^{\mu}\left(\mathbb{M} \times \mathbb{R}, \boldsymbol{g} ; N_{-}, N_{+}\right)_{(\ell)}$ be (anisotropic) elliptic. Then there exists a $\mathcal{P} \in \mathcal{C}^{-\mu}\left(\mathbb{M} \times \mathbb{R}, \boldsymbol{g}^{-1} ; N_{+}, N_{-}\right)_{(\ell)}$ for $\boldsymbol{g}^{-1}=(\gamma-\mu, \gamma, \Theta ; \delta, \Xi)$ such that $\mathcal{P} \mathcal{A}=\mathcal{I}+\mathcal{C}_{l}$, $\mathcal{A P}=\mathcal{I}+\mathcal{C}_{r}$ for certain $\mathcal{C}_{l} \in \mathcal{C}^{-\infty}\left(\mathbb{M} \times \mathbb{R}, \boldsymbol{g}_{r} ; N_{-}, N_{-}\right), \mathcal{C}_{r} \in \mathcal{C}^{-\infty}\left(\mathbb{M} \times \mathbb{R}, \boldsymbol{g}_{l} ; N_{+}, N_{+}\right)$for $\boldsymbol{g}_{l}=(\gamma, \gamma, \Theta ; \delta, \Xi), \boldsymbol{g}_{r}=(\gamma-\mu, \gamma-\mu, \Theta ; \delta, \Xi)$.

Proof. We can construct $\mathcal{P}$ in the form $\mathcal{P}=\sum_{j=0}^{1} \varphi_{j} \mathcal{P}_{j} \psi_{j}$ for any choice of functions $\varphi_{j}, \psi_{j} \in C^{\infty}(\mathbb{R})$ such that $\sum_{j=0}^{1} \varphi_{j}=1, \varphi_{j} \psi_{j}=\varphi_{j}$ for $j=0,1$, such that $\varphi_{0}, \psi_{0}$ are equal to 1 for $t \leq t_{0}$ and 0 for $t \geq \widetilde{t}_{0}$, for certain $t_{0}<\widetilde{t}_{0}$, and $\varphi_{1}, \psi_{1}$ are equal to 1 for $t \geq t_{1}$ and 0 for $t \leq \widetilde{t}_{1}$ for certain $\widetilde{t}_{1}<t_{1}$. The operators $\mathcal{P}_{j}$ can be chosen as parametrices of the anisotropic elliptic edge operator $\mathcal{A}$, applying the scheme of the isotropic theory, cf. [19], Section 3.5.2. By virtue of the assumptions on the coefficients the construction of $\mathcal{P}_{0}$ can be carried out uniformly up to $t=-\infty$. This step has some relation to a corresponding result of Seiler [24] in its anisotropic analogue. [24] contains many other useful elements of the edge operator technique (cf. Gil, Schulze, and Seiler [4]). The operator $\mathcal{P}_{1}$ can be constructed along the lines of the proof of the parametrix part of Theorem 3.3.4 from [15], again a in corresponding anisotropic form. An inspection of the proof shows that the discrete limits for $t \rightarrow \infty$ of asymptotic types in the occurring operators remain preserved in all steps. The reason is the particular nature of the inverse of the principal conormal corner symbol, cf. Remark 3.3.2. Applying the compatibility of the constructions on every finite $t$-interval we get $\mathcal{P}$ in the asserted form.

Theorem 3.3.4 Let $\mathcal{A} \in \mathcal{C}^{\mu}\left(\mathbb{M} \times \mathbb{R}, \boldsymbol{g} ; N_{-}, N_{+}\right)_{(\ell)}$ be (anisotropic) elliptic. Then $u \in$ $\mathcal{W}^{-\infty, \gamma ; \delta}(\mathbb{M} \times \mathbb{R})_{(\ell)} \oplus H^{-\infty ; \delta}\left(Y, \mathbb{C}^{N_{-}}\right)$and

$$
\mathcal{A} u=f \in \mathcal{W}_{\widetilde{P}, \widetilde{Q}}^{s-\mu, \gamma-\mu ; \delta}(\mathbb{M} \times \mathbb{R})_{(\ell)} \oplus H_{\widetilde{R}}^{(s-\mu) / \ell ; \delta}\left(Y, \mathbb{C}^{N_{+}}\right)
$$


for any $s \in \mathbb{R}$ and asymptotic types $(\widetilde{P}, \widetilde{Q} ; \widetilde{R})$ implies $u \in \mathcal{W}_{P, Q}^{s, \gamma ; \delta}(\mathbb{M} \times \mathbb{R})_{(\ell)} \oplus H_{R}^{s / \ell ; \delta}\left(Y, \mathbb{C}^{N_{-}}\right)$ for certain resulting asymptotic types $(P, Q ; R)$. If $(\widetilde{P}, \widetilde{Q} ; \widetilde{R})$ have discrete limits for $t \rightarrow \infty$, then the same is true of $(P, Q ; R)$.

Proof. Applying Theorem 3.3.3 we find a parametrix $\mathcal{P} \in \mathcal{C}^{-\mu}\left(\mathbb{M} \times \mathbb{R}, \boldsymbol{g}^{-1} ; N_{+}, N_{-}\right)_{(\ell)}$ of $\mathcal{A}$, such that, in particular, $\mathcal{P} \mathcal{A} u=u+\mathcal{C}_{l} u$ for every $u \in \mathcal{W}^{-\infty, \gamma ; \delta}(\mathbb{M} \times \mathbb{R})_{(\ell)} \oplus$ $H^{-\infty ; \delta}\left(Y, \mathbb{C}^{N_{-}}\right)$, where $\mathcal{C}_{l} \in \mathcal{C}^{-\infty}\left(\mathbb{M} \times \mathbb{R}, \boldsymbol{g}_{r} ; N_{-}, N_{-}\right)$. The fact that $\mathcal{P}$ acts as a continuous operator between spaces with asymptotics (cf. formula (3.2.3)) gives us $\mathcal{P} \mathcal{A} u \in$ $\mathcal{W}_{P_{1}, Q_{1}}^{s, \gamma ; \delta}(\mathbb{M} \times \mathbb{R})_{(\ell)} \oplus H_{R_{1}}^{s / \ell ; \delta}\left(Y, \mathbb{C}^{N_{-}}\right)$for certain asymptotic types $\left(P_{1}, Q_{1} ; R_{1}\right)$. Moreover, we have $\mathcal{C}_{l} u \in \mathcal{W}_{P_{2}, Q_{2}}^{\infty, \gamma ; \delta}(\mathbb{M} \times \mathbb{R}) \oplus H_{R_{2}}^{\infty ; \delta}\left(Y, \mathbb{C}^{N_{-}}\right)$for other asymptotic types $\left(P_{2}, Q_{2} ; R_{2}\right)$ (cf. formula (3.2.2)). This yields altogether $u=\mathcal{P} \mathcal{A} u-\mathcal{C}_{l} u \in\left\{\mathcal{W}_{P_{1}, Q_{1}}^{s, \gamma ; \delta}(\mathbb{M} \times \mathbb{R})_{(\ell)} \oplus\right.$ $\left.H_{R_{1}}^{s / \ell ; \delta}\left(Y, \mathbb{C}^{N_{-}}\right)\right\}+\left\{\mathcal{W}_{P_{2}, Q_{2}}^{\infty, \gamma ; \delta}(\mathbb{M} \times \mathbb{R}) \oplus H_{R_{2}}^{\infty ; \delta}\left(Y, \mathbb{C}^{N_{-}}\right)\right\}$which is the desired result, since the latter space is contained in a space of the form $\mathcal{W}_{P, Q}^{s, \gamma ; \delta}(\mathbb{M} \times \mathbb{R})_{(\ell)} \oplus H_{R}^{s / \ell ; \delta}\left(Y, \mathbb{C}^{N_{-}}\right)$for a suitable choice of $(P, Q ; R)$. In this construction the asymptotic types $\left(P_{i}, Q_{i} ; R_{i}\right), i=1,2$, and $(P, Q ; R)$ have discrete limits for $t \rightarrow \infty$ if this property is assumed on $(\widetilde{P}, \widetilde{Q} ; \widetilde{R})$, because compositions of operators in our algebra as well as continuous maps in corresponding weighted Sobolev spaces with asymptotics preserve discrete limits for $t \rightarrow \infty$.

Remark 3.3.5 Our constructions become simpler if we consider operators with constant coefficients for $t>T$ for some $T$. In that case we may choose the edge part of the asymptotics of $f$ to be constant and discrete for $t>T$, and we then get the same for $u$ for large $t$.

\section{References}

[1] M.S. Agranovich and M.I. Vishik, Elliptic problems with parameter and parabolic problems of general form, Russ. Math. Surv. 19, 3 (1964), 53-157.

[2] L. Boutet de Monvel, Boundary problems for pseudo-differential operators, Acta Math. 126 (1971), 11-51.

[3] T. Buchholz and B.-W. Schulze, Anisotropic edge pseudo-differential operators with discrete asymptotics, Math. Nachr. 184 (1997), 73-125.

[4] J.B. Gil, B.-W Schulze, and J. Seiler, Cone pseudo-differential operators in the edge symbolic calculus, Osaka J. Math. 37 (2000), 219-258.

[5] V.A. Kondrat'ev, Boundary value problems for elliptic equations in domains with conical points, Trudy Mosk. Mat. Obshch. 16 (1967), 209-292.

[6] T. Krainer, Parabolic pseudodifferential operators and long-time asymptotics of solutions, Ph.D. thesis, University of Potsdam, 2000.

[7] T. Krainer and B.-W. Schulze, Long-time asymptotics for parabolic operators in the pseudodifferential cone algebra approach, Preprint 99/12, Institute for Mathematics, Potsdam, 1999.

[8] V.G. Maz'ja and B.A. Plamenevskij, On the asymptotic behaviour of solutions of differential equations in Hilbert space, Math. USSR Izvestia 6 (1972), 1067-1116.

[9] A. Pazy, Asymptotic expansions of solutions of ordinary differential equations in Hilbert space, Arch. Ration. Mech. Anal. 24 (1967), 193-218. 
[10] S. Rempel and B.-W. Schulze, Index theory of elliptic boundary problems, AkademieVerlag, Berlin, 1982.

[11] B.-W. Schulze, Ellipticity and continuous conormal asymptotics on manifolds with conical singularities, Math. Nachr. 136 (1988), 7-57.

[12] B.-W. Schulze, Regularity with continuous and branching asymptotics for elliptic operators on manifolds with edges, Integral Equations Operator Theory 11 (1988), 557-602.

[13] B.-W. Schulze, Pseudo-differential operators on manifolds with edges, Symposium "Partial Differential Equations", Holzhau 1988, Teubner-Texte zur Mathematik, vol. 112, Teubner, Leipzig, 1989, pp. 259-287.

[14] B.-W. Schulze, Pseudo-differential operators on manifolds with singularities, NorthHolland, Amsterdam, 1991.

[15] B.-W. Schulze, The Mellin pseudo-differential calculus on manifolds with corners, Symposium: "Analysis in Domains and on Manifolds with Singularities", Breitenbrunn 1990, Teubner-Texte zur Mathematik, vol. 131, Teubner, Leipzig, 1992, pp. 208-289.

[16] B.-W. Schulze, Pseudo-differential boundary value problems, conical singularities, and asymptotics, Akademie Verlag, Berlin, 1994.

[17] B.-W. Schulze, The variable discrete asymptotics in pseudo-differential boundary value problems I, Advances in Partial Differential Equations (Pseudo-Differential Calculus and Mathematical Physics), Akademie Verlag, Berlin, 1994, pp. 9-96.

[18] B.-W. Schulze, The variable discrete asymptotics in pseudo-differential boundary value problems II, Advances in Partial Differential Equations (Boundary Value Problems, Schrödinger Operators, Deformation Quantization), Akademie Verlag, Berlin, 1995, pp. 9-69.

[19] B.-W. Schulze, Boundary value problems and singular pseudo-differential operators, J. Wiley, Chichester, 1998.

[20] B.-W. Schulze, Operator algebras with symbol hierarchies on manifolds with singularities, Preprint 99/30, Institute for Mathematics, Potsdam, 1999.

[21] B.-W. Schulze, Iterated asymptotics on corner manifolds, Proceedings PDE Conference Wuhan, April 5-9, 1999, World Scientific Publishing Co. of Singapore, 2000.

[22] B.-W. Schulze, Operators on corner manifolds, J. Wiley, (in preparation).

[23] B.-W. Schulze and I. Witt, The variable discrete asymptotics on manifolds with edge singularities, Preprint, Institute for Mathematics, Potsdam, 1999, (in preparation).

[24] J. Seiler, Pseudodifferential calculus on manifolds with non-compact edges, Ph.D. thesis, University of Potsdam, 1998.

[25] J. Seiler, Continuity of edge and corner pseudo-differential operators, Math. Nachr. 205 (1999), 163-182. 